\title{
NEW TESTS OF CALENDAR EFFECTS ON EQUITY AND SECURITIZED REAL ESTATE MARKETS
}

\author{
Eddie C. M. HUI ${ }^{1^{*}}$, Ka Kwan Kevin CHAN ${ }^{1}$ \\ ${ }^{1}$ Department of Building and Real Estate, The Hong Kong Polytechnic University, Hong Kong, China
}

Received 21 November 2017; accepted 30 April 2018

\begin{abstract}
We construct two new tests of calendar effects, apply them on 12 stock indices during 1996-2016, and compare the results with that using Hui and Chan (2016)'s method. The results show that the January and Halloween effects are significant for the four western generalized equity indices for small moving-window sizes. Furthermore, the securitized real estate indices show a greater difference in the overall calendar effect between the three methods than the general equity indices do. This study has an implication that a certain sector of the market is riskier than the whole market.
\end{abstract}

Keywords: calendar effect, Shiryaev-Zhou index, moving-window size, trading strategy, smoothing effect.

\section{Introduction}

Many investors adhere to the "buy-and-hold" strategy backed by the efficient market hypothesis (EMH), which is supported by a lot of articles (e.g. Malkiel \& Fama, 1970; Barber \& Odean, 2000). However, after the global financial crisis in 2008, many scholars raised doubts on the EMH (Hui \& Chan, 2018a). If the EMH does not hold, then "buy-and-hold" may not work. Monthly trends in stock price movement called "calendar effects" are found in many previous studies, with the Halloween and January effects being the most common ones. The former says that equities get significantly higher returns during NovemberApril, and is first discovered by Bouman and Jacobsen (2002), who find much greater average stock returns in November-April during 1970-1998. The January effect states that equity returns are significantly higher in January, and is first found by Wachtel (1942) on high-yields stocks in the U.S.

Most of the previous studies on calendar effects use traditional methods like regression. Hui and Chan (2016) apply Shiryaev-Zhou index of variable moving-window size to test calendar effects of 12 stock indices during 1996-2014. The Shiryaev-Zhou index of a stock, commonly denoted by $\mu$, is calculated by dividing the stock's annual drift (or return) by its annual volatility (or variance of return). Deducting $1 / 2$ from the quotient results in the Shiryaev-Zhou index (see Section 3). It can be regarded as a benchmark value for trading stock as follows: buy and hold the stock until the end of the period if $\mu \geq 0$, and sell it immediately otherwise (Yam et al., 2012a, 2012b). From the Shiryaev-Zhou index, Hui et al. (2014b) derive the estimator of the Shiryaev-Zhou index $\hat{\mu}_{i}(n)$ (they fix the moving-window size $n$ to be 130) and introduce a trading strategy as follows: buy a stock as soon as $\hat{\mu}_{i}(n)$ changes from negative to positive, and sell it as soon as $\hat{\mu}_{i}(n)$ changes from positive to negative. Hui and Chan (2017) extend their work by letting the moving-window size $n$ to vary, deriving a generalized time-dependent strategy.

The method of Hui and Chan (2016) is based on Hui and Chan (2017)'s generalized time-dependent strategy of which one has to buy a stock (or stock index) as soon as the estimator of the Shiryaev-Zhou index $\hat{\mu}_{i}(n)$ changes from negative to positive, and sell it as soon as $\hat{\mu}_{i}(n)$ changes from positive to negative. The recent globalization has strengthened interrelationship between international financial markets, increasing their volatility. Hence stock prices fluctuate a lot and $\hat{\mu}_{i}(n)$ changes sign very frequently. Thus, we have to trade the stock frequently. Since $\hat{\mu}_{i}(n)$ lags behind the stock price (Hui \& Chan, 2014), there is a chance that on day $i$, the stock price is rising even when $\hat{\mu}_{i}(n)$ is negative, so "buy-and-hold" would outperform the strategy. In reality, transaction costs exist and build up for the strategy. When the amount of transaction costs increases, there is a higher chance that the strategy underperforms "buy-and-hold" (Hui \& Chan, 2014). In fact, some studies even find that the strategy

*Corresponding author. E-mail: bscmhui@polyu.edu.hk 
underperforms "buy-and-hold" for the majority of the cases (see Section 1). In light of this, Hui and Chan (2018c) construct two alternative strategies of which after $\hat{\mu}_{i}(n)$ changes sign, an investor waits until $\hat{\mu}_{i}(n)$ remains at the same sign for two or three consecutive days before buying or selling the stock index (which are called Strategy 2 and Strategy 3 respectively in Hui and Chan, 2018a, see details in Section 3). This reduces the number of times of trading the stock index and is called "smoothing effect". Their results show that Strategy 3 outperforms the other two strategies in general, especially when transaction costs exist.

In this study, based on Hui and Chan (2018c)'s three trading strategies, we construct three tests (Methods 1, 2 and 3 (see Section 4)). Method 1 is the test by Hui and Chan, 2016, while Methods 2 and 3 are newly constructed) to investigate calendar effects of 12 stock indices during 1996-2016. In particular, the Halloween and January effects are investigated. We follow Hui and Chan (2016)'s method: the overall calendar effect is examined by analysis of mean (ANOM), and the Halloween effect is tested by logistic regression. We compare the results of the three methods based on the three trading strategies, so we can see that for each of the three strategies, whether the stock index is held for most of the time in a particular month. Since Hui and Chan (2018c)'s two alternative strategies are different from Hui and Chan (2017)'s strategy, the "holding periods" and "non-holding periods" are different. Hence we expect that the resulting calendar effects shown by the three strategies are also different. One of the contributions of our study is that our new tests of calendar effects (Methods 2 and 3) can show different patterns of calendar effects from that shown by Hui and Chan (2016)'s method. We also compare the results between western (US, UK, France and Germany) and Asian markets (Hong Kong and Japan), and between securitized real estate and general equity indices. Hence investors can see different patterns of calendar effects between different types of markets. This is useful for them to improve their trading strategies to increase their profits.

The article continues as follows: Section 1 wraps up previous works. Section 2 presents the data source. Section 3 describes the formula of the Shiryaev-Zhou index and its estimator, and list out Hui and Chan (2018c)'s three trading strategies. The tests of calendar effects are described in Section 4. Section 5 displays and analyzes the results. Last section concludes the article.

\section{Literature review}

There are plenty of studies on calendar effects, especially the Halloween and January effects. Tables 1 and 2 (Hui \& Chan, 2016) summarize previous works.

Table 1. Results of previous works on Halloween Effect (source: Hui \& Chan, 2015, 2016)

\begin{tabular}{|c|c|c|c|c|c|}
\hline Article & Method & Countries and regions & Asset types & $\begin{array}{c}\text { Period of } \\
\text { observation }\end{array}$ & $\begin{array}{l}\text { Result (significant } \\
\text { or insignificant) }\end{array}$ \\
\hline $\begin{array}{l}\text { Sullivan, } \\
\text { Timmermann, } \\
\text { and White } \\
(2001)\end{array}$ & Bootstrap & U.S. & Equity & $1897-1996$ & Insignificant \\
\hline $\begin{array}{l}\text { Bouman and } \\
\text { Jacobsen }(2002)\end{array}$ & Linear regression & $\begin{array}{l}\text { Australia, Austria, Belgium, Canada, } \\
\text { Denmark, France, Germany, Hong } \\
\text { Kong, Italy, Japan, Netherlands, } \\
\text { Norway, Singapore, Spain, Sweden, } \\
\text { Switzerland, U.K., U.S., Argentina, } \\
\text { Brazil, Chile, Finland, Greece, } \\
\text { Indonesia, Ireland, Jordan, Malaysia, } \\
\text { Mexico, New Zealand, Philippines, } \\
\text { Portugal, Russia, South Africa, } \\
\text { South Korea, Taiwan, Thailand, } \\
\text { Turkey }\end{array}$ & Equity & $1970-1998$ & $\begin{array}{l}\text { Significant (except } \\
\text { for one country) }\end{array}$ \\
\hline $\begin{array}{l}\text { Lucey and } \\
\text { Whelan (2002) }\end{array}$ & Out-of-sample test & U.S., U.K., Ireland & Equity & 1934-1999 & Significant \\
\hline $\begin{array}{l}\text { Maberly and } \\
\text { Pierce (2004) }\end{array}$ & Linear regression & U.S. & Equity & $1970-1998$ & Insignificant \\
\hline $\begin{array}{l}\text { Brounen and } \\
\text { Hamo (2009) }\end{array}$ & Linear regression & $\begin{array}{l}\text { U.S., Japan, Hong Kong, U.K., } \\
\text { Australia, France, Singapore, } \\
\text { Canada, Netherlands, Austria, South } \\
\text { Africa }\end{array}$ & Equity & $1987-2007$ & $\begin{array}{l}\text { Significant for } 5 \\
\text { countries }\end{array}$ \\
\hline $\begin{array}{l}\text { Jacobsen and } \\
\text { Visaltanachoti } \\
(2009)\end{array}$ & Linear regression & U.S. & Equity & $1926-2006$ & Significant \\
\hline Lean (2011) & $\operatorname{GARCH}(1,1)$ & $\begin{array}{l}\text { Hong Kong, China, Japan, } \\
\text { Singapore, Malaysia, India }\end{array}$ & Equity & $1991-2008$ & Significant \\
\hline
\end{tabular}


End of Table 1

\begin{tabular}{|c|c|c|c|c|c|}
\hline Article & Method & Countries and regions & Asset types & $\begin{array}{c}\text { Period of } \\
\text { observation }\end{array}$ & $\begin{array}{l}\text { Result (significant } \\
\text { or insignificant) }\end{array}$ \\
\hline $\begin{array}{l}\text { Andrade, } \\
\text { Chhaochharia, } \\
\text { and Fuerst } \\
(2012)\end{array}$ & $\begin{array}{l}\text { Linear regression, } \\
\text { out-of-sample test }\end{array}$ & $\begin{array}{l}\text { Australia, Austria, Belgium, Canada, } \\
\text { Denmark, France, Germany, Hong } \\
\text { Kong, Italy, Japan, Netherlands, Nor- } \\
\text { way, Singapore, Spain, Sweden, Swit- } \\
\text { zerland, U.K., U.S., Argentina, Brazil, } \\
\text { Chile, Finland, Greece, Indonesia, } \\
\text { Ireland, Jordan, Malaysia, Mexico, } \\
\text { New Zealand, Philippines, Portugal, } \\
\text { Russia, South Africa, South Korea, } \\
\text { Taiwan, Thailand, Turkey }\end{array}$ & Equity & $1970-2012$ & Significant \\
\hline $\begin{array}{l}\text { Hui, Wright, and } \\
\text { Yam (2014a) }\end{array}$ & $\begin{array}{l}\text { Linear regression, } \\
\text { White's Reality } \\
\text { Check and } \\
\text { Hansen's Superior } \\
\text { Ability tests }\end{array}$ & $\begin{array}{l}\text { Canada, U.S., Hong Kong, Japan, } \\
\text { Philippines, Singapore, Belgium, } \\
\text { Finland, France, Germany, Italy, } \\
\text { Netherlands, Sweden, Switzerland, } \\
\text { U.K., Australia }\end{array}$ & $\begin{array}{l}\text { Real Estate } \\
\text { (including } \\
\text { REIT) }\end{array}$ & $1984-2011$ & $\begin{array}{l}\text { Linear regression: } \\
\text { significant for two } \\
\text { countries only. } \\
\text { White's Reality } \\
\text { Check and Hansen's } \\
\text { Superior Ability } \\
\text { tests: insignificant }\end{array}$ \\
\hline
\end{tabular}

Table 2. Results of previous works on January Effect (source: Hui \& Chan, 2015, 2016)

\begin{tabular}{|c|c|c|c|c|c|}
\hline Article & Method & Countries and regions & Asset types & $\begin{array}{l}\text { Period of } \\
\text { observation }\end{array}$ & $\begin{array}{l}\text { Result (significant or } \\
\text { insignificant) }\end{array}$ \\
\hline Keim (1983) & Linear regression & U.S. & Equity & $1963-1979$ & Significant \\
\hline $\begin{array}{l}\text { Agrawal and } \\
\text { Tandon (1994) }\end{array}$ & Linear regression & $\begin{array}{l}\text { Australia, Belgium, Brazil, Canada, } \\
\text { Denmark, France, Germany, Hong } \\
\text { Kong, Italy, Japan, Luxembourg, Mex- } \\
\text { ico, Netherlands, New Zealand, Singa- } \\
\text { pore, Sweden, Switzerland, U.K., U.S. }\end{array}$ & Equity & $1971-1987$ & $\begin{array}{l}\text { Significant for } 10 \\
\text { countries }\end{array}$ \\
\hline $\begin{array}{l}\text { Cheung and } \\
\text { Coutts (1999) }\end{array}$ & Linear regression & Hong Kong & Equity & $1985-1997$ & Insignificant \\
\hline $\begin{array}{l}\text { Fountas and } \\
\text { Segredakis } \\
(2002)\end{array}$ & Linear regression & $\begin{array}{l}\text { Argentina, Chile, Colombia, Greece, } \\
\text { India, Jordan, Korea, Malaysia, Mex- } \\
\text { ico, Nigeria, Pakistan, Philippines, } \\
\text { Portugal, Taiwan, Thailand, Turkey, } \\
\text { Venezuela, Zimbabwe }\end{array}$ & Equity & $1987-1995$ & $\begin{array}{l}\text { Insignificant (except for } \\
\text { Chile) }\end{array}$ \\
\hline Gu (2003) & $\begin{array}{l}\text { Power ratio } \\
\text { method }\end{array}$ & U.S. & Equity & $1929-2000$ & $\begin{array}{l}\text { Declining (becoming } \\
\text { less significant over } \\
\text { time) }\end{array}$ \\
\hline $\begin{array}{l}\text { Hansen, Lunde, } \\
\text { and Nason } \\
(2005)\end{array}$ & $\rho$ test & $\begin{array}{l}\text { Denmark, France, Germany, Hong } \\
\text { Kong, Italy, Japan, Norway, Sweden, } \\
\text { U.K., U.S. }\end{array}$ & Equity & $1896-2002$ & Significant \\
\hline $\begin{array}{l}\text { Hardin III, } \\
\text { Liano, and } \\
\text { Huang (2005) }\end{array}$ & Linear regression & U.S. & REIT & $1994-2002$ & $\begin{array}{l}\text { Insignificant for the } \\
\text { REIT value-weighted } \\
\text { index, but significant } \\
\text { for the REIT equal- } \\
\text { weighted index }\end{array}$ \\
\hline $\begin{array}{l}\text { Brounen and } \\
\text { Hamo (2009) }\end{array}$ & Linear regression & $\begin{array}{l}\text { U.S., Japan, Hong Kong, U.K., Austra- } \\
\text { lia, France, Singapore, Canada, The } \\
\text { Netherlands, Austria, South Africa }\end{array}$ & Equity & $1987-2007$ & Insignificant \\
\hline $\begin{array}{l}\text { Kang, Jiang, Lee, } \\
\text { and Yoon (2010) }\end{array}$ & Linear regression & China & Equity & $1996-2007$ & $\begin{array}{l}\text { A-share: insignificant } \\
\text { B-share: significant } \\
\text { at } 10 \% \text { level, but } \\
\text { insignificant at } 5 \% \text { level }\end{array}$ \\
\hline $\begin{array}{l}\text { Almudhaf and } \\
\text { Hansz (2011) }\end{array}$ & Linear regression & $\begin{array}{l}\text { Belgium, Denmark, Finland, France, } \\
\text { Germany, Italy, Netherlands, Norway, } \\
\text { Spain, Sweden, Switzerland, U.K. }\end{array}$ & $\begin{array}{l}\text { Securitized } \\
\text { real estate }\end{array}$ & $1990-2007$ & $\begin{array}{l}\text { Insignificant (except for } \\
\text { Switzerland) }\end{array}$ \\
\hline Hui et al. (2014a) & $\begin{array}{l}\text { Linear regression, } \\
\text { White's Reality } \\
\text { Check and } \\
\text { Hansen's Superior } \\
\text { Ability tests }\end{array}$ & $\begin{array}{l}\text { Canada, U.S., Hong Kong, Japan, } \\
\text { Philippines, Singapore, Belgium, } \\
\text { Finland, France, Germany, Italy, } \\
\text { Netherlands, Sweden, Switzerland, } \\
\text { U.K., Australia }\end{array}$ & $\begin{array}{l}\text { Real Estate } \\
\text { (including } \\
\text { REIT) }\end{array}$ & $1984-2011$ & Insignificant \\
\hline
\end{tabular}


While the previous studies listed in the above tables contribute to knowledge by testing the significance of Halloween and January effects of various markets, they produce mixed results. For Halloween effect, the majority of previous works show that the effect is significant, but some others find insignificant Halloween effect (see Table 1). The results of January effect are even more diverged: about half of the studies find significant January effect, while others show that the effect is insignificant (see Table 2). The majority of the articles work on equity markets. Only a sparse number of them investigate real estate markets. Thus, the true pattern of calendar effects of property markets is still unknown. Furthermore, most of the previous studies apply traditional methods like linear regression. Only a few of them use alternative tests. Different methods may lead to different results. For example, Hui et al. (2014a) examine calendar effects of 27 securitized real estate indices from 20 countries. Applying linear regression, statistically significant calendar anomalies persist. However, no calendar rule outperforms the "buy-and-hold" strategy significantly according to the White's Reality Check (White, 2000) and Hansen's Superior Predictive Ability (Hansen, 2005) tests.

Recently, some studies apply Shiryaev-Zhou index to investigate calendar effects. Hui and Chan (2015) apply Shiryaev-Zhou index to examine the January and Halloween effects of 8 securitized property markets, fixing the moving-window size to be 130 days. Therefore, the resulting strategy may not be optimal. Furthermore, we cannot see how the calendar effect varies as the moving-window size increases or decreases. Therefore, Hui and Chan (2016) apply Shiryaev-Zhou index of variable movingwindow size to test calendar effects of 12 stock indices. However, as mentioned in introduction, $\hat{\mu}_{i}(n)$ changes sign frequently, so we have to trade the stock/stock index for many times for the underlying trading strategy. Hui and Chan (2018a) test this underlying strategy on 12 stock indices, and find that their strategy outperforms "buy-andhold" for only slight majority of the cases. Hui and Chan (2018b) apply the same strategy on 12 Hong Kong listed stocks. They even find that the strategy underperforms "buy-and-hold" for slight majority of the cases. In light of this, this study introduces two new tests of calendar effects based on Hui and Chan (2018c)'s two newly constructed strategies of which one waits until $\hat{\mu}_{i}(n)$ remains at the same sign for two or three consecutive days before buying or selling the stock/stock index (which are called Strategy 2 and Strategy 3 respectively in Hui and Chan, 2018c). This leads to a "smoothing effect", reducing the number of times of trading the stock/stock index and yields a greater profit than the original strategy (which is called Strategy 1 in Hui and Chan, 2018c). Hence we can see that for Strategies 2 and 3, whether we should hold the stock/stock index for the majority of the time in a particular month. This is useful for investors to improve their trading strategies to earn more profits.

\section{Data}

The whole period is 1 January 1996-31 December 2016, a total of 5480 observations. The period is traced back by 240 days (the maximum moving-window size) to 28 January 1995. We select one securitized real estate index and one general equity index for each of the six economies: Hong Kong, Japan, U.S., U.K., France and Germany. The 12 stock indices in Table 3 are chosen. The reason for choosing the 12 stock indices is explained in Hui and Chan (2018b).

Table 3. The stock indices we choose

\begin{tabular}{|l|l|l|}
\hline Economy & \multicolumn{1}{|c|}{$\begin{array}{c}\text { General equity } \\
\text { index }\end{array}$} & Securitized real estate index \\
\hline $\begin{array}{l}\text { Hong } \\
\text { Kong }\end{array}$ & $\begin{array}{l}\text { Hang Seng Index } \\
\text { (HSI) }\end{array}$ & $\begin{array}{l}\text { FTSE EPRA/NAREIT Hong } \\
\text { Kong Index (ELHK) }\end{array}$ \\
\hline Japan & $\begin{array}{l}\text { Nikkei 225 Index } \\
\text { (NKY) }\end{array}$ & $\begin{array}{l}\text { FTSE EPRA/NAREIT Japan } \\
\text { Index (ELJP) }\end{array}$ \\
\hline U.S. & $\begin{array}{l}\text { S\&P 500 Index } \\
\text { (SPX) }\end{array}$ & $\begin{array}{l}\text { FTSE EPRA/NAREIT US } \\
\text { Index (UNUS) }\end{array}$ \\
\hline U.K. & $\begin{array}{l}\text { FTSE 100 Index } \\
\text { (UKX) }\end{array}$ & $\begin{array}{l}\text { FTSE EPRA/NAREIT UK } \\
\text { Index (ELUK) }\end{array}$ \\
\hline France & $\begin{array}{l}\text { CAC 40 Index } \\
\text { (CAC) }\end{array}$ & $\begin{array}{l}\text { FTSE EPRA/NAREIT France } \\
\text { Index (EPFR) }\end{array}$ \\
\hline Germany & DAX Index (DAX) & $\begin{array}{l}\text { FTSE EPRA/NAREIT } \\
\text { Germany Index (EPGR) }\end{array}$ \\
\hline
\end{tabular}

\section{The Shiryaev-Zhou index and the trading strategies}

The Shiryaev-Zhou index comes from. Shiryaev, Xu, and Zhou (2008), who solve the problem of minimizing the time between a stock's maximum and selling prices by deriving the "goodness index" of a stock. Du Toit and Peskir (2008) provide a probabilistic proof of the solution. Yam, Yam, Yung, and Zhou (2009, 2012a, 2012b) resolve the same problem in the binomial tree setting and generalize the Shiryaev-Zhou index over the corresponding framework. Hui et al. (2014a) derive a trading strategy from the Shiryaev-Zhou index and apply it on four western securitized real estate indices and six Asian securitized real estate indices respectively. Both of them find that their strategy outperforms "buy-and-hold" in general.

The Shiryaev-Zhou index is described by the following formula (Yam et al., 2009, 2012a, 2012b; Hui et al., 2014a; Hui \& Chan, 2018c):

$$
\mu=\left(\alpha-0.5 \sigma^{2}\right) / \sigma^{2}=\alpha / \sigma^{2}-0.5,
$$

where: $\alpha$ is the annual drift (or return) of the stock; and $\sigma$ is its annual volatility (or variance of return).

In reality, the values of $\alpha, \sigma$ are always varying and their exact values are normally not known. Therefore, we adopt the moving-window approach by Wong et al. (2012) to estimate their values, and obtain the estimator of the Shiryaev-Zhou index (Hui \& Chan, 2016, 2018a, 2018b):

$$
\hat{\mu}_{i}(n)=\frac{\hat{\alpha}_{i}(n)-0.5 \hat{\sigma}_{i}^{2}(n)}{\hat{\sigma}_{i}^{2}(n)},
$$


where: $n$ denotes the moving-window size; $\hat{\alpha}_{i}(n)$ and $\hat{\sigma}_{i}^{2}(n)$ are the estimators of $\alpha$ and $\sigma^{2}$ on day $i$ respectively.

As in Hui and Chan (2016), we choose the following 6 moving-window sizes: 40, 80, 120, 160, 200, 240.

Hui and Chan (2018c) apply $\hat{\mu}_{i}(n)$ to derive three trading strategies. We make the following assumptions:

(1) We make the transaction price of a stock index to be its closing price.

(2) The amount of cash held at the start of the period is enough to cover all transactions during the period.

Denote the final day of the period by day $N$. The first strategy is called Strategy 1 (Hui \& Chan, 2018b):

1. On day 1 , if $\hat{\mu}_{1}(n) \geq 0$, buy 1 unit of the stock index. No action is taken otherwise.

2. From day 2 to day $N-1$, adopt the following rule:

(a) if $\hat{\mu}_{i-1}(n) \geq 0$ and $\hat{\mu}_{i}(n) \geq 0$, no action is taken.

(b) if $\hat{\mu}_{i-1}(n) \geq 0$ and $\hat{\mu}_{i}(n)<0$, sell the entire 1 unit of the stock index we hold.

(c) if $\hat{\mu}_{i-1}(n)<0$ and $\hat{\mu}_{i}(n) \geq 0$, buy 1 unit of the stock index.

(d) if $\hat{\mu}_{i-1}(n)<0$ and $\hat{\mu}_{i}(n)<0$, no action is taken.

3. On day $N$, if 1 unit of the stock index is still held,

sell all of it. Otherwise, no action is taken.

According to Hui and Chan (2018c), since $\hat{\mu}_{i}(n)$ changes sign very frequently, one has to trade the stock index for a lot of times when he/she adopts Strategy 1, so this strategy may underperform "buy-and-hold", especially when transaction costs exist. Therefore, Hui and Chan (2018c) construct two new trading strategies of which we wait until $\hat{\mu}_{i}(n)$ remains at the same sign for a number of consecutive days before changing our buying/selling position. For first strategy, after $\hat{\mu}_{i}(n)$ changes sign, an investor waits until the sign of $\hat{\mu}_{i}(n)$ remains unchanged for 2 consecutive days, then he/she buy or sell the stock index. This is called Strategy 2 (Hui \& Chan, 2018c):

1. On day 1 , if $\hat{\mu}_{1}(n) \geq 0$, buy 1 unit of the stock index. No action is taken otherwise.

2. On day 2 , no action is taken.

3. From day 3 to day $N-1$ (denote that day by day $i$ ):

(a) If one is holding entire cash, then buy 1 unit of the stock index if $\hat{\mu}_{i}(n)>=0$ on days $i-1$ and $i$. Otherwise, no action is taken.

(b) If one is holding 1 unit of the stock index, then sell all of it if $\hat{\mu}_{i}(n)<0$ on days $i-1$ and $i$. Otherwise, no action is taken.

4. On day $N$, if 1 unit of the stock index is still held, sell all of it. Otherwise, no action is taken.

For the second strategy, after $\hat{\mu}_{i}(n)$ changes sign, an investor waits until the sign of $\hat{\mu}_{i}(n)$ remains unchanged for 3 consecutive days, then he/she buy or sell the stock index. This is called Strategy 3 (Hui \& Chan, 2018a):

1. On day 1 , if $\hat{\mu}_{1}(n) \geq 0$, buy 1 unit of the stock index. No action is taken otherwise.

2. On both day 2 and day 3, do not take any action.

3. From day 4 to day $N-1$ (denote that day by day $i$ ):

(c) If one is holding entire cash, then buy 1 unit of the stock index if $\hat{\mu}_{i}(n)>=0$ on days $i-2, i-1$ and $i$. Otherwise, no action is taken. (d) If one is holding 1 unit of the stock index, then sell all of it if $\hat{\mu}_{i}(n)<0$ on days $i-2, i-1$ and $i$. Otherwise, no action is taken.

4. On day $N$, if 1 unit of the stock index is still held, sell all of it. Otherwise, no action is taken.

Hui and Chan (2018a) test Strategies 1, 2 and 3 on the 12 stock indices chosen in Section 2 during the period December 29, 1995 - December 31, 2016. They find that Strategies 2 and 3 outperform Strategy 1 in overall. In particular, Strategy 3 earns the maximum profit out of the three strategies, and hence is the best strategy. Since Strategies 1, 2 and 3 are different, their "holding periods" (periods of which the stock index is held according to Strategies 1, 2 or 3) and "non-holding periods" (periods of which entire cash is held according to Strategies 1, 2 or 3) are also different. Therefore, our tests (see Section 4) based on the three strategies will result in different patterns of calendar effects as shown in Section 5.

\section{Testing the calendar effects}

We construct three tests of calendar effect according the Hui and Chan (2018c)'s three trading strategies. For the first method (the method by Hui and Chan, 2016, denoted by Method 1), the Halloween and January effects can be represented by the following hypotheses:

$H_{11}$ (Halloween effect): the proportion of time of which the stock index is held according to Strategy 1 is significantly higher (i.e. the "holding period" of Strategy 1 is significantly longer) during November - April. (null hypothesis: $\left.H_{10}\right)$.

$J_{11}$ (January Effect): the proportion of time of which the stock index is held according to Strategy 1 is significantly higher (i.e. the "holding period" of Strategy 1 is significantly longer) in January. (null hypothesis: $J_{10}$ ).

We adopt Hui and Chan (2016)'s method to test the overall calendar effect: for each moving-window size $n$ and each stock index, define a dummy variable $R_{1 i}$ by $R_{1 i}=1$ when $\hat{\mu}_{i-1}(n) \geq 0$ (i.e. the stock index is held according to Strategy 1 on day $i$ ), and 0 otherwise. Hence the period of which $R_{1 i}=1$ is called the "holding period" of Strategy 1, while the period of which $R_{1 i}=0$ is called the "non-holding period" of Strategy 1. Therefore, the average value of $R_{1 i}$ in each month indicates the percentage of time of which the stock index is held in that month according to Strategy 1. The overall calendar effect (and hence the January effect) is tested using analysis of mean (ANOM). The details of ANOM are described in Hui and Chan (2016). To examine the overall calendar effect, we use the software Minitab 17 to perform a one-way ANOM of $R_{1 i}$ with month as the factor.

For the second method (denoted by Method 2), the Halloween and January effects can be represented by the following hypotheses:

$H_{21}$ (Halloween effect): the proportion of time of which the stock index is held according to Strategy 2 is significantly higher (i.e. the "holding period" of Strategy 2 is significantly longer) during November - April. (null hypothesis: $H_{20}$ ). 
$J_{21}$ (January Effect): the proportion of time of which the stock index is held according to Strategy 2 is significantly higher (i.e. the "holding period" of Strategy 2 is significantly longer) in January. (null hypothesis: $J_{20}$ ).

We also apply ANOM to test the overall calendar effect as in Method 1. However, the dummy variable $R_{1 i}$ is replaced by $R_{2 i}$, which is defined by $R_{2 i}=1$ if the stock index is held according to Strategy 2 on day $i$, and 0 otherwise. Hence the period of which $R_{2 i}=1$ is called the "holding period" of Strategy 2, while the period of which $R_{2 i}=0$ is called the "non-holding period" of Strategy 2. The average value of $R_{2 i}$ in each month indicates the percentage of time of which the stock index is held in that month according to Strategy 2.

For the third method (denoted by Method 3), the Halloween and January effects can be represented by the following hypotheses:

$H_{31}$ (Halloween effect): the proportion of time of which the stock index is held according to Strategy 3 is significantly higher (i.e. the "holding period" of Strategy 3 is significantly longer) during November - April. (null hypothesis: $H_{30}$ ).

$J_{31}$ (January Effect): the proportion of time of which the stock index is held according to Strategy 3 is significantly higher (i.e. the "holding period" of Strategy 3 is significantly longer) in January. (null hypothesis: $J_{30}$ ).

As in Methods 1 and 2, we apply ANOM to test the overall calendar effect. However, we use a new dummy variable $R_{3 i}$ which is defined by $R_{3 i}=1$ if the stock index is held according to Strategy 3 on day $i$, and 0 otherwise. Hence the period of which $R_{3 i}=1$ is called the "holding period" of Strategy 3, while the period of which $R_{3 i}=0$ is called the "non-holding period" of Strategy 3. The average value of $R_{3 i}$ in each month indicates the percentage of time of which the stock index is held in that month according to Strategy 3.

For the normal method, we use ANOM, too, but $R_{1 i}$ is replaced by the continuously compounded daily return $r_{i}=\log \left(\frac{S_{i}}{S_{i-1}}\right)$, where $S_{i}$ is the stock index on day $i$.

We apply regression to test the Halloween effect. However, since the dependent variables $R_{1 i}, R_{2 i}$ and $R_{3 i}$ are dummy variables which have values of either 0 or 1 , linear regression is inappropriate. Instead, logistic regression is applied. For Method 1, the following model is set up (Hui \& Chan, 2016):

$$
\operatorname{logit}\left(E\left(R_{1 i} \mid D_{i}\right)\right)=\alpha+\beta D_{i}+\varepsilon_{i},
$$

where: $\operatorname{logit}(x)=x /(1-x), E(X)$ represents the expected value of $X ; D_{i}$ is a dummy variable which is equal to 1 when day $i$ is in the period November - April, and 0 otherwise. Logistic regression is applied to (4.1), and a one-tailed z-test is conducted on $\frac{\hat{\beta}}{\hat{\sigma}}$, where $\hat{\beta}$ is the estimator of $\beta$, and $\hat{\sigma}$ is the standard deviation of $\hat{\beta}$. Again, we use Minitab 17 to perform logistic regression.

For Methods 2 and 3, the model is same as (4.1), but the dependent variable $R_{1 i}$ is replaced by $R_{2 i}$ and $R_{3 i}$ respectively.
For the normal method, we apply the following linear regression model (Hui \& Chan, 2016):

$$
r_{i}=\gamma+\lambda D_{i}+\varepsilon_{i}
$$

where: $r_{i}=\log \left(\frac{S_{i}}{S_{i-1}}\right)$ and $D_{i}$ is the $\hat{\lambda}_{\hat{\lambda}}$ variable in (4.1). A one-tailed z-test is conducted on $\frac{\hat{\lambda}}{\hat{\varphi}}$, where $\hat{\lambda}$ is the OLS estimator of $\lambda$, and $\hat{\varphi}$ is the standard deviation of $\hat{\lambda}$.

Methods 1, 2 and 3 are based on Strategies 1,2 and 3 respectively. The dummy variables $R_{1 i}, R_{2 i}$ and $R_{3 i}$ indicate the "holding periods" (i.e. periods of which we should hold the stock index according to the strategy) of Strategies 1, 2 and 3 respectively. The difference between the dummy variables $R_{1 i}, R_{2 i}$ and $R_{3 i}$ results in the difference between the pattern of calendar effects shown by Methods 1, 2 and 3. Hence this study is different from Hui and Chan (2016)'s work.

Method 1 by Hui and Chan (2016) can show the proportion of time of which the stock index is held in each month according to Strategy 1, and Hui and Chan (2016) find that this method can show additional channels of calendar effects. However, Hui and Chan (2018c) find that Strategy 1 underperforms "buy-and-hold" for slight majority of the cases. In light of this, Hui and Chan (2018c) construct two new strategies (Strategies 2 and 3 in this study). They show that the two strategies, especially Strategy 3, outperform Strategy 1 and "buy-and-hold" in general. Since Methods 2 and 3 can show the proportion of time of which the stock index is held in each month according to Strategies 2 and 3 (which are superior to Strategy 1 according to Hui and Chan, 2018c) respectively, they are superior to Strategy 1, and can help investors to improve their strategies to increase their profits.

\section{Results}

\subsection{Overall calendar effect and January effect}

We use the methods described in Section 4 to examine the calendar effects of the 12 stock indices. Firstly, we apply ANOM to test the overall calendar effect and January effect. We list out the results in the Tables 4, 5, 6 and 7.

The entries in Tables 4, 5, 6 and 7 indicate the mean value of $\log \left(\frac{S_{i}}{S_{i-1}}\right), R_{1 i}, R_{2 i}$ and $R_{3 i}$ of that index in the corresponding month for the corresponding movingwindow size, respectively. The green/red entries indicate that they lie above/below the $95 \%$ confidence interval respectively. For Tables 5, 6 and 7, the green entries mean that the "holding periods" of Strategies 1, 2 and 3, respectively, are significantly longer at $5 \%$ level, while the red entries mean that the "holding periods" of Strategies 1, 2 and 3, respectively, are significantly shorter at 5\% level. In particular, the entries in the column January indicate whether the January effect is significant at $5 \%$ level. Green entries imply that the January effect is significant at $5 \%$ level, while red entries mean that the January effect goes into reverse, and the effect is significant at $5 \%$ level. 
Table 4. The test results for the overall calendar effect using the normal method

\begin{tabular}{|c|c|c|c|c|c|c|c|c|c|c|c|c|c|}
\hline Index & Jan & Feb & Mar & Apr & May & Jun & Jul & Aug & Sep & Oct & Nov & Dec & $\begin{array}{c}95 \% \\
\text { confidence } \\
\text { interval }\end{array}$ \\
\hline HSI & -0.000771 & 0.000959 & -0.000801 & 0.001112 & -0.000370 & 0.000130 & 0.000996 & -0.000816 & -0.000158 & 0.000532 & 0.000876 & 0.000468 & $\begin{array}{c}(-0.0020 \\
0.0023)\end{array}$ \\
\hline $\mathrm{NKY}$ & -0.000149 & 000336 & 0.000681 & 0.000832 & -0.000624 & 0.000793 & -0.000712 & -0.001013 & -0.000246 & -0.000969 & 0.000459 & 0.000441 & $\begin{array}{c}(-0.0018 \\
0.0018)\end{array}$ \\
\hline SPX & -0.000004 & -0.000253 & 0.000746 & 0.000945 & & & & -0.000481 & & 0.000707 & 0.000769 & & $\begin{array}{c}(-0.0014 \\
0.0019)\end{array}$ \\
\hline UKX & -0.000547 & 0.000408 & 0.000168 & 0.000964 & -0.000335 & -0.000685 & 0.000317 & -0.000123 & -0.000643 & 0.000633 & 0.000340 & 0.000896 & $\begin{array}{c}(-0.0015 \\
0.0017)\end{array}$ \\
\hline CAC & 0.000229 & 0.000062 & 0.000647 & 0.001103 & -0.000358 & -0.000196 & -0.000099 & -0.000702 & -0.000936 & 0.000735 & 0.000657 & 0.000838 & $\begin{array}{c}(-0.0018, \\
0.0021)\end{array}$ \\
\hline DAX & 0.000167 & 0.000057 & \begin{tabular}{|l|}
0.000466 \\
\end{tabular} & 0.001527 & 0.000096 & 0.000131 & 0.000278 & -0.001433 & -0.001083 & 0.000967 & 0.001259 & 0.001106 & $\begin{array}{c}(-0.0017, \\
0.0023)\end{array}$ \\
\hline
\end{tabular}

\begin{tabular}{|c|c|c|c|c|c|c|c|c|c|c|c|c|c|}
\hline Index & Jan & Feb & Mar & Apr & May & Jun & Jul & Aug & Sep & Oct & Nov & Dec & $\begin{array}{c}95 \% \\
\text { confidence } \\
\text { interval }\end{array}$ \\
\hline ELHK & -0.000015 & 0.000612 & -0.000582 & 0.001148 & -0.001003 & -0.000445 & 0.001501 & -0.000322 & -0.001010 & 0.000484 & 0.001167 & 0.001158 & $\begin{array}{c}(-0.0024 \\
0.0028)\end{array}$ \\
\hline ELJP & 0.000403 & 0.000654 & 0.001409 & 0.001184 & -0.000329 & 0.000411 & -0.000766 & -0.000293 & 0.000414 & -0.000065 & -0.000733 & -0.000352 & $\begin{array}{c}(-0.0026 \\
0.0029)\end{array}$ \\
\hline UNUS & -0.000097 & -0.000453 & 0.000866 & 0.001409 & 0.000168 & -0.000198 & 0.000487 & 0.000052 & -0.000029 & -0.000553 & -0.000260 & 0.001210 & $\begin{array}{c}(-0.0021, \\
0.0025)\end{array}$ \\
\hline ELUK & -0.000500 & 0.000761 & 0.000044 & 0.001087 & 0.000181 & -0.001262 & 0.000739 & 0.000569 & -0.000604 & -0.000161 & -0.000296 & 0.000684 & $\begin{array}{c}(-0.0016 \\
0.0018)\end{array}$ \\
\hline EPFR & 0.000569 & 0.001313 & 0.000754 & 0.000138 & 0.000347 & -0.000717 & 0.000562 & | 0.000308 & 0.000059 & 0.000147 & -0.000122 & 0.000807 & $\begin{array}{c}(-0.0013, \\
0.0020)\end{array}$ \\
\hline EPGR & 0.000617 & 0.000249 & 0.000015 & 0.000985 & 0.000376 & -0.001658 & 0.000125 & -0.000057 & -0.001394 & 0.000676 & -0.000913 & 0.000650 & $\begin{array}{c}(-0.0022, \\
0.0021)\end{array}$ \\
\hline
\end{tabular}

Table 5. The test results for the overall calendar effect using Method 1

\begin{tabular}{|c|c|c|c|c|c|c|c|c|c|c|c|c|c|}
\hline \multicolumn{14}{|c|}{ HSI } \\
\hline $\begin{array}{l}\text { Moving- } \\
\text { window } \\
\text { size }\end{array}$ & Jan & Feb & Mar & Apr & May & Jun & Jul & Aug & Sep & Oct & Nov & Dec & $\begin{array}{c}95 \% \\
\text { confidence } \\
\text { interval }\end{array}$ \\
\hline 40 & 0.5376 & 0.4706 & 0.4147 & 0.5066 & 0.6099 & 0.4722 & 0.5739 & 0.6523 & 0.5765 & 0.5386 & 0.6362 & 0.5996 & $\begin{array}{l}(0.4872 \\
0.6120)\end{array}$ \\
\hline 80 & 0.6946 & 0.5953 & 0.4881 & 0.4580 & 0.5172 & 0.4588 & 0.5032 & 0.5400 & 0.6297 & 0.5837 & 0.5938 & 0.5867 & $\begin{array}{l}(0.4917 \\
0.6164)\end{array}$ \\
\hline 120 & 0.6213 & 0.5647 & 0.6134 & 0.5863 & 0.4892 & 0.4610 & 0.5246 & 0.4838 & 0.6009 & 0.6052 & 0.6763 & 0.6467 & $\begin{array}{l}(0.5100 \\
0.6342)\end{array}$ \\
\hline 160 & 0.6688 & 0.6424 & 0.5680 & 0.6615 & 0.5970 & 0.5479 & 0.5268 & 0.5745 & 0.5299 & 0.5579 & 0.6205 & 0.6188 & $\begin{array}{l}(0.5306 \\
0.6545) \\
\end{array}$ \\
\hline 200 & 0.6258 & 0.6118 & 0.6264 & 0.6549 & 0.6056 & 0.5768 & 0.6445 & 0.6220 & 0.6452 & 0.5815 & 0.5781 & 0.6103 & $\begin{array}{l}(0.5538 \\
0.6769) \\
\end{array}$ \\
\hline 240 & 0.5419 & 0.5718 & 0.5724 & 0.6084 & 0.6358 & 0.6347 & 0.5717 & 0.6566 & 0.6608 & 0.6288 & 0.5960 & 0.6039 & $\begin{array}{l}(0.5452, \\
0.6686)\end{array}$ \\
\hline \multicolumn{14}{|c|}{ NKY } \\
\hline $\begin{array}{c}\text { Moving- } \\
\text { window size }\end{array}$ & Jan & Feb & Mar & Apr & May & Jun & Jul & Aug & Sep & Oct & Nov & Dec & $\begin{array}{l}95 \% \text { con- } \\
\text { fidence } \\
\text { interval }\end{array}$ \\
\hline 40 & 0.5505 & 0.5388 & 0.5572 & 0.6018 & 0.5539 & 0.4766 & 0.5482 & 0.4384 & 0.3614 & 0.4356 & 0.5134 & 0.5418 & $\begin{array}{l}\text { (0.4471, } \\
0.5726)\end{array}$ \\
\hline 80 & 0.5699 & 0.5435 & 0.6436 & 0.5841 & 0.5366 & 0.5479 & 0.5525 & 0.3499 & 0.3636 & 0.3433 & 0.4174 & 0.4625 & $\begin{array}{l}(0.4306 \\
0.5548)\end{array}$ \\
\hline 120 & 0.5054 & 0.4988 & 0.6156 & 0.6150 & 0.5366 & 0.5278 & 0.5782 & 0.4492 & 0.3459 & 0.2876 & 0.4018 & 0.4582 & $\begin{array}{l}(0.4230 \\
0.5471)\end{array}$ \\
\hline 160 & 0.4989 & 0.6776 & 0.5421 & 0.6018 & 0.5022 & 0.5612 & 0.5161 & 0.4600 & 0.4523 & 0.4206 & 0.3237 & 0.4004 & $\begin{array}{l}(0.4171 \\
0.5424)\end{array}$ \\
\hline 200 & 0.3978 & 0.4471 & 0.5637 & 0.4403 & 0.4612 & 0.5056 & 0.5824 & 0.5421 & 0.4812 & 0.4292 & 0.4487 & 0.4347 & $\begin{array}{l}(0.4152 \\
0.5410)\end{array}$ \\
\hline 240 & 0.5118 & 0.4706 & 0.4320 & 0.4602 & 0.4397 & 0.4143 & 0.4754 & 0.4816 & 0.4922 & 0.5215 & 0.5067 & 0.4861 & $\begin{array}{l}(0.4113 \\
0.5376)\end{array}$ \\
\hline
\end{tabular}


Continue of Table 5

\begin{tabular}{|c|c|c|c|c|c|c|c|c|c|c|c|c|c|}
\hline \multicolumn{14}{|c|}{ SPX } \\
\hline $\begin{array}{l}\text { Moving- } \\
\text { window } \\
\text { size }\end{array}$ & Jan & Feb & Mar & Apr & May & Jun & Jul & Aug & Sep & Oct & Nov & Dec & $\begin{array}{l}95 \% \text { con- } \\
\text { fidence } \\
\text { interval }\end{array}$ \\
\hline 40 & 0.6817 & 0.5694 & 0.6328 & 0.6748 & 0.6918 & 0.5412 & 0.5846 & 0.4816 & 0.5188 & 0.5408 & 0.7321 & 0.7537 & $\begin{array}{l}(0.5567 \\
0.6779)\end{array}$ \\
\hline 80 & 0.7806 & 0.7153 & 0.6847 & 0.6836 & 0.7112 & 0.6012 & 0.6317 & 0.5054 & 0.5721 & 0.5021 & 0.5580 & 0.7880 & $\begin{array}{c}(0.5858 \\
0.7047)\end{array}$ \\
\hline 120 & 0.7011 & 0.7082 & 0.8272 & 0.7478 & 0.6765 & 0.6815 & 0.7002 & 0.6328 & 0.5499 & 0.5472 & 0.6674 & 0.6702 & $\begin{array}{c}(0.6179 \\
0.7350)\end{array}$ \\
\hline 160 & 0.6753 & 0.6659 & 0.7063 & 0.7721 & 0.8297 & 0.7684 & 0.7516 & 0.6717 & 0.6851 & 0.5773 & 0.6384 & 0.6617 & $\begin{array}{l}(0.6429 \\
0.7578)\end{array}$ \\
\hline 200 & 0.6753 & 0.6565 & 0.7149 & 0.7080 & 0.7457 & 0.7951 & 0.7837 & 0.7689 & 0.7273 & 0.6416 & 0.7031 & 0.7238 & $\begin{array}{l}(0.6641 \\
0.7772)\end{array}$ \\
\hline 240 & 0.6710 & 0.6965 & 0.7171 & 0.7013 & 0.7069 & 0.7149 & 0.7837 & 0.7797 & 0.7694 & 0.7275 & 0.7321 & 0.7323 & $\begin{array}{c}(0.6717 \\
0.7841)\end{array}$ \\
\hline \multicolumn{14}{|c|}{ UKX } \\
\hline $\begin{array}{l}\text { Moving- } \\
\text { window } \\
\text { size }\end{array}$ & Jan & Feb & Mar & Apr & May & Jun & Jul & Aug & Sep & Oct & Nov & Dec & $\begin{array}{l}\text { 95\% con- } \\
\text { fidence } \\
\text { interval }\end{array}$ \\
\hline 40 & 0.6839 & 0.6141 & 0.6048 & 0.6327 & 0.6530 & 0.4076 & 0.4861 & 0.5011 & 0.5521 & 0.5601 & 0.6942 & 0.6317 & $\begin{array}{l}(0.5235 \\
0.6466)\end{array}$ \\
\hline 80 & 0.7462 & 0.6965 & 0.6177 & 0.6925 & 0.6875 & 0.4922 & 0.4839 & 0.4060 & 0.5166 & 0.5172 & 0.5603 & 0.6895 & $\begin{array}{l}(0.5310 \\
0.6526)\end{array}$ \\
\hline 120 & 0.6366 & 0.6565 & 0.7775 & 0.6858 & 0.6638 & 0.5768 & 0.5632 & 0.4536 & 0.4989 & 0.5365 & 0.5714 & 0.5760 & $\begin{array}{l}(0.5383 \\
0.6606)\end{array}$ \\
\hline 160 & 0.5763 & 0.5765 & 0.6609 & 0.7389 & 0.7457 & 0.6548 & 0.6424 & 0.5659 & 0.6075 & 0.5386 & 0.5402 & 0.5525 & $\begin{array}{c}(0.5558 \\
0.6777)\end{array}$ \\
\hline 200 & 0.5785 & 0.5576 & 0.6523 & 0.6261 & 0.6681 & 0.6904 & 0.7516 & 0.6264 & 0.6896 & 0.6030 & 0.6228 & 0.5782 & $\begin{array}{c}(0.5769 \\
0.6979) \\
\end{array}$ \\
\hline 240 & 0.6323 & 0.6165 & 0.5918 & 0.5863 & 0.6164 & 0.6012 & 0.6724 & 0.6739 & 0.6918 & 0.6438 & 0.6808 & 0.6617 & $\begin{array}{l}(0.5793, \\
0.7006)\end{array}$ \\
\hline \multicolumn{14}{|c|}{ CAC } \\
\hline $\begin{array}{l}\text { Moving- } \\
\text { window } \\
\text { size }\end{array}$ & Jan & Feb & Mar & Apr & May & Jun & Jul & Aug & Sep & Oct & Nov & $\mathrm{Dec}$ & $\begin{array}{l}95 \% \text { con- } \\
\text { fidence } \\
\text { interval }\end{array}$ \\
\hline 40 & 0.6860 & 0.6494 & 0.5788 & 0.6991 & 0.6746 & 0.5033 & 0.4325 & 0.4557 & 0.5809 & 0.5773 & 0.6161 & 0.6403 & $\begin{array}{l}(0.5293 \\
0.6520)\end{array}$ \\
\hline 80 & 0.7269 & 0.6706 & 0.6998 & 0.7124 & 0.6573 & 0.5768 & 0.5096 & 0.4104 & 0.5078 & 0.5193 & 0.5290 & 0.6188 & $\begin{array}{l}(0.5335, \\
0.6555)\end{array}$ \\
\hline 120 & 0.5441 & 0.6824 & 0.7646 & 0.7279 & 0.6616 & 0.5635 & 0.5803 & 0.5205 & 0.5011 & 0.4850 & 0.5491 & 0.5396 & $\begin{array}{l}(0.5315 \\
0.6539)\end{array}$ \\
\hline 160 & 0.5570 & 0.6094 & 0.6436 & 0.7146 & 0.7069 & 0.5947 & 0.6381 & 0.6004 & 0.6475 & 0.4979 & 0.5022 & 0.5332 & $\begin{array}{l}(0.5423, \\
0.6650)\end{array}$ \\
\hline 200 & 0.5806 & 0.6141 & 0.6177 & 0.6195 & 0.6379 & 0.6325 & 0.6531 & 0.6112 & 0.7007 & 0.6738 & 0.6585 & 0.5931 & $\begin{array}{c}(0.5717 \\
0.6936) \\
\end{array}$ \\
\hline 240 & 0.6387 & 0.6518 & 0.6264 & 0.6659 & 0.6487 & 0.6392 & 0.6188 & 0.6458 & 0.6829 & 0.6481 & 0.6897 & 0.6702 & $\begin{array}{l}(0.5917 \\
0.7123)\end{array}$ \\
\hline \multicolumn{14}{|c|}{ DAX } \\
\hline $\begin{array}{l}\text { Moving- } \\
\text { window } \\
\text { size }\end{array}$ & Jan & Feb & Mar & Apr & May & Jun & Jul & Aug & Sep & Oct & Nov & Dec & $\begin{array}{l}95 \% \text { con- } \\
\text { fidence } \\
\text { interval }\end{array}$ \\
\hline 40 & 0.7591 & 0.6635 & 0.5853 & 0.6637 & 0.6616 & 0.5301 & 0.5075 & 0.4665 & 0.4967 & 0.5515 & 0.6741 & 0.7645 & $\begin{array}{l}(0.5497 \\
0.6708)\end{array}$ \\
\hline 80 & 0.7677 & 0.7365 & 0.6845 & 0.6836 & 0.6810 & 0.5969 & 0.5739 & 0.4514 & 0.5366 & 0.5043 & 0.5558 & 0.6959 & $\begin{array}{l}(0.5617 \\
0.6821)\end{array}$ \\
\hline 120 & 0.5871 & 0.6541 & 0.7603 & 0.7611 & 0.7392 & 0.6815 & 0.6745 & 0.5659 & 0.5632 & 0.5322 & 0.5960 & 0.6017 & $\begin{array}{l}(0.5830 \\
0.7028)\end{array}$ \\
\hline 160 & 0.6086 & 0.6165 & 0.6479 & 0.7190 & 0.7974 & 0.7283 & 0.7409 & 0.6436 & 0.6541 & 0.5751 & 0.6339 & 0.6595 & $\begin{array}{l}(0.6099 \\
0.7281)\end{array}$ \\
\hline 200 & 0.6430 & 0.6165 & 0.6220 & 0.6173 & 0.6509 & 0.7149 & 0.7859 & 0.7214 & 0.7162 & 0.6137 & 0.6585 & 0.6702 & $\begin{array}{l}(0.6103 \\
0.7287)\end{array}$ \\
\hline 240 & 0.6068 & 0.6659 & 0.6220 & 0.6173 & 0.6185 & 0.6214 & 0.6595 & 0.7149 & 0.7694 & 0.7082 & 0.7478 & 0.7366 & $\begin{array}{l}(0.6229 \\
0.7402)\end{array}$ \\
\hline
\end{tabular}


Continue of Table 5

\begin{tabular}{|c|c|c|c|c|c|c|c|c|c|c|c|c|c|}
\hline \multicolumn{14}{|c|}{ ELHK } \\
\hline $\begin{array}{l}\text { Moving- } \\
\text { window } \\
\text { size }\end{array}$ & Jan & Feb & Mar & Apr & May & Jun & Jul & Aug & Sep & Oct & Nov & Dec & $\begin{array}{l}95 \% \text { con- } \\
\text { fidence } \\
\text { interval }\end{array}$ \\
\hline 40 & 0.6172 & 0.4424 & 0.4384 & 0.4469 & 0.5819 & 0.4588 & 0.5567 & 0.6177 & 0.5965 & 0.5043 & 0.5245 & 0.5310 & $\begin{array}{l}0.4645, \\
0.5899)\end{array}$ \\
\hline 80 & 0.6258 & 0.5929 & 0.4989 & 0.5111 & 0.4892 & 0.3920 & 0.4540 & 0.5205 & 0.6231 & 0.6438 & 0.5692 & 0.5203 & $\begin{array}{l}(0.4740, \\
0.5990)\end{array}$ \\
\hline 120 & 0.6473 & 0.5765 & 0.5680 & 0.6128 & 0.5733 & 0.5078 & 0.4518 & 0.5054 & 0.6098 & 0.5515 & 0.6629 & 0.6531 & $\begin{array}{l}(0.5144, \\
0.6385)\end{array}$ \\
\hline 160 & 0.6602 & 0.6471 & 0.5616 & 0.5155 & 0.5754 & 0.5078 & 0.5289 & 0.5788 & 0.5965 & 0.5901 & 0.5982 & 0.6274 & $\begin{array}{l}(0.5199, \\
0.6443)\end{array}$ \\
\hline 200 & 0.5871 & 0.6141 & 0.6393 & 0.6084 & 0.5797 & 0.4900 & 0.5246 & 0.5680 & 0.6231 & 0.6073 & 0.5982 & 0.5953 & $\begin{array}{l}(0.5239, \\
0.6483)\end{array}$ \\
\hline 240 & 0.5785 & 0.5929 & 0.5572 & 0.6460 & 0.6250 & 0.5702 & 0.5696 & 0.6501 & 0.6541 & 0.6373 & 0.5915 & 0.6103 & $\begin{array}{l}0.5452, \\
0.6687) \\
\end{array}$ \\
\hline \multicolumn{14}{|c|}{ ELJP } \\
\hline $\begin{array}{l}\text { Moving- } \\
\text { window } \\
\text { size }\end{array}$ & Jan & Feb & Mar & Apr & May & Jun & Jul & Aug & Sep & Oct & Nov & Dec & $\begin{array}{l}95 \% \text { con- } \\
\text { fidence } \\
\text { interval }\end{array}$ \\
\hline 40 & 0.4774 & 0.5365 & 0.6264 & 0.6836 & 0.5474 & 0.4143 & 0.5139 & 0.4039 & 0.4102 & 0.5687 & 0.5201 & 0.3897 & $\begin{array}{l}(0.4452 \\
0.5698)\end{array}$ \\
\hline 80 & 0.4753 & 0.4447 & 0.5292 & 0.6239 & 0.6746 & 0.5969 & 0.4411 & 0.3521 & 0.3592 & 0.3734 & 0.4487 & 0.4388 & $\begin{array}{l}0.4178, \\
0.5417) \\
\end{array}$ \\
\hline 120 & 0.4237 & 0.4447 & 0.5076 & 0.5597 & 0.5711 & 0.5835 & 0.6424 & 0.5594 & 0.3836 & 0.4034 & 0.4174 & 0.4026 & $\begin{array}{l}(0.4296, \\
0.5544)\end{array}$ \\
\hline 160 & 0.4473 & 0.3835 & 0.4816 & 0.5619 & 0.5409 & 0.5145 & 0.5867 & 0.6285 & 0.5831 & 0.5408 & 0.3571 & 0.3597 & $\begin{array}{l}(0.4373, \\
0.5620)\end{array}$ \\
\hline 200 & 0.4215 & 0.4071 & 0.4773 & 0.4292 & 0.5453 & 0.5612 & 0.5503 & 0.5896 & 0.5255 & 0.5901 & 0.6049 & 0.4711 & $\begin{array}{l}(0.4522, \\
0.5777)\end{array}$ \\
\hline 240 & 0.5247 & 0.5035 & 0.4471 & 0.4270 & 0.4397 & 0.4543 & 0.5931 & 0.5659 & 0.4789 & 0.5644 & 0.5089 & 0.5032 & $\begin{array}{l}(0.4383, \\
0.5643)\end{array}$ \\
\hline \multicolumn{14}{|c|}{ UNUS } \\
\hline $\begin{array}{l}\text { Moving- } \\
\text { window } \\
\text { size }\end{array}$ & Jan & Feb & Mar & Apr & May & Jun & Jul & Aug & Sep & Oct & Nov & Dec & $\begin{array}{l}95 \% \text { con- } \\
\text { fidence } \\
\text { interval }\end{array}$ \\
\hline 40 & 0.7462 & 0.6118 & 0.6069 & 0.5907 & 0.6250 & 0.5390 & 0.5782 & 0.6264 & 0.5032 & 0.4700 & 0.4933 & 0.6017 & $\begin{array}{l}(0.5212, \\
0.6448)\end{array}$ \\
\hline 80 & 0.7011 & 0.6682 & 0.7214 & 0.7301 & 0.6983 & 0.6904 & 0.6167 & 0.5918 & 0.5654 & 0.5129 & 0.4107 & 0.5396 & $\begin{array}{l}(0.5601, \\
0.6807)\end{array}$ \\
\hline 120 & 0.6000 & 0.6871 & 0.7473 & 0.8031 & 0.7780 & 0.7884 & 0.7088 & 0.6091 & 0.6142 & 0.5172 & 0.5379 & 0.5396 & $\begin{array}{l}(0.6018, \\
0.7190) \\
\end{array}$ \\
\hline 160 & 0.6000 & 0.5929 & 0.7041 & 0.7655 & 0.8168 & 0.7906 & 0.6981 & 0.6393 & 0.6231 & 0.5837 & 0.5335 & 0.5460 & $\begin{array}{l}0.5991, \\
0.7170) \\
\end{array}$ \\
\hline 200 & 0.6215 & 0.6141 & 0.6955 & 0.7212 & 0.7953 & 0.8018 & 0.7816 & 0.7214 & 0.6585 & 0.5794 & 0.5759 & 0.6017 & $\begin{array}{l}(0.6229, \\
0.7392)\end{array}$ \\
\hline 240 & 0.6280 & 0.6635 & 0.6566 & 0.6814 & 0.7543 & 0.7127 & 0.7816 & 0.6911 & 0.6874 & 0.6803 & 0.5960 & 0.5910 & $\begin{array}{l}(0.6184, \\
0.7360)\end{array}$ \\
\hline \multicolumn{14}{|c|}{ ELUK } \\
\hline $\begin{array}{l}\text { Moving- } \\
\text { window } \\
\text { size }\end{array}$ & Jan & Feb & Mar & Apr & May & Jun & Jul & Aug & Sep & Oct & Nov & Dec & $\begin{array}{l}95 \% \text { con- } \\
\text { fidence } \\
\text { interval }\end{array}$ \\
\hline 40 & 0.6323 & 0.7247 & 0.6048 & 0.5929 & 0.6875 & 0.4833 & 0.4454 & 0.6091 & 0.6031 & 0.4506 & 0.5335 & 0.4325 & $\begin{array}{l}(0.5038, \\
0.6272)\end{array}$ \\
\hline 80 & 0.6000 & 0.6118 & 0.6674 & 0.6615 & 0.7198 & 0.6102 & 0.6231 & 0.5875 & 0.4967 & 0.5966 & 0.5491 & 0.4946 & $\begin{array}{l}(0.5401, \\
0.6631)\end{array}$ \\
\hline 120 & 0.5763 & 0.5294 & 0.6134 & 0.6261 & 0.6961 & 0.7261 & 0.6188 & 0.5572 & 0.5610 & 0.5536 & 0.5513 & 0.6317 & $\begin{array}{l}(0.5423, \\
0.6654)\end{array}$ \\
\hline 160 & 0.5978 & 0.5835 & 0.6026 & 0.6084 & 0.6703 & 0.6860 & 0.7024 & 0.6566 & 0.6075 & 0.5858 & 0.5491 & 0.5203 & $\begin{array}{l}(0.5531, \\
0.6757)\end{array}$ \\
\hline 200 & 0.6108 & 0.6118 & 0.6069 & 0.6659 & 0.6465 & 0.6682 & 0.6296 & 0.6652 & 0.6319 & 0.5974 & 0.6138 & 0.5803 & $\begin{array}{l}(0.5645, \\
0.6869)\end{array}$ \\
\hline 240 & 0.6237 & 0.6306 & 0.6199 & 0.6261 & 0.6681 & 0.6526 & 0.5663 & 0.6026 & 0.5765 & 0.5494 & 0.5893 & 0.5760 & $\begin{array}{l}(0.5445, \\
0.6679)\end{array}$ \\
\hline
\end{tabular}


End of Table 5

\begin{tabular}{|c|c|c|c|c|c|c|c|c|c|c|c|c|c|}
\hline \multicolumn{14}{|c|}{ EPFR } \\
\hline $\begin{array}{l}\text { Moving- } \\
\text { window } \\
\text { size }\end{array}$ & Jan & Feb & Mar & Apr & May & Jun & Jul & Aug & Sep & Oct & Nov & Dec & $\begin{array}{l}95 \% \text { con- } \\
\text { fidence } \\
\text { interval } \\
\end{array}$ \\
\hline 40 & 0.6645 & 0.6824 & 0.7538 & 0.6770 & 0.6142 & 0.4855 & 0.4647 & 0.5421 & 0.5543 & 0.5386 & 0.5647 & 0.5096 & $\begin{array}{l}(0.5256 \\
0.6485)\end{array}$ \\
\hline 80 & 0.6817 & 0.6471 & 0.7063 & 0.8319 & 0.7931 & 0.5857 & 0.5096 & 0.5227 & 0.4989 & 0.5451 & 0.5156 & 0.5567 & $\begin{array}{l}(0.5560, \\
0.6761)\end{array}$ \\
\hline 120 & 0.6538 & 0.6965 & 0.8121 & 0.7677 & 0.7953 & 0.7439 & 0.6809 & 0.6134 & 0.5233 & 0.4292 & 0.4799 & 0.5739 & $\begin{array}{l}(0.5887, \\
0.7058)\end{array}$ \\
\hline 160 & 0.5548 & 0.6729 & 0.6911 & 0.7633 & 0.7845 & 0.7216 & 0.7388 & 0.6998 & 0.6430 & 0.5730 & 0.5692 & 0.5246 & $\begin{array}{l}(0.6021, \\
0.7201)\end{array}$ \\
\hline 200 & 0.6280 & 0.6000 & 0.6523 & 0.6836 & 0.7220 & 0.7617 & 0.7752 & 0.7581 & 0.7494 & 0.7039 & 0.6518 & 0.6253 & $\begin{array}{l}(0.6351, \\
0.7510)\end{array}$ \\
\hline 240 & 0.7290 & 0.6871 & 0.6955 & 0.6527 & 0.7543 & 0.7528 & 0.7902 & 0.7991 & 0.8226 & 0.7017 & 0.7455 & 0.7216 & $\begin{array}{l}(0.6826, \\
0.7933)\end{array}$ \\
\hline \multicolumn{14}{|c|}{ EPGR } \\
\hline $\begin{array}{l}\text { Moving- } \\
\text { window } \\
\text { size }\end{array}$ & Jan & Feb & Mar & Apr & May & Jun & Jul & Aug & Sep & Oct & Nov & Dec & $\begin{array}{l}95 \% \text { con- } \\
\text { fidence } \\
\text { interval }\end{array}$ \\
\hline 40 & 0.5075 & 0.5976 & 0.5616 & 0.5044 & 0.6379 & 0.5212 & 0.4133 & 0.5356 & 0.5632 & 0.4206 & 0.5223 & 0.4176 & $\begin{array}{l}(0.4533, \\
0.5788)\end{array}$ \\
\hline 80 & 0.4839 & 0.4353 & 0.6436 & 0.6350 & 0.6724 & 0.5590 & 0.4839 & 0.5140 & 0.4302 & 0.4807 & 0.5134 & 0.4475 & $\begin{array}{c}(0.4629, \\
0.5878)\end{array}$ \\
\hline 120 & 0.5118 & 0.4776 & 0.5918 & 0.5730 & 0.7091 & 0.6169 & 0.5246 & 0.5443 & 0.4967 & 0.5086 & 0.5022 & 0.5482 & $\begin{array}{l}(0.4884, \\
0.6134)\end{array}$ \\
\hline 160 & 0.5075 & 0.5529 & 0.6285 & 0.5642 & 0.6358 & 0.6102 & 0.5953 & 0.5594 & 0.5188 & 0.5794 & 0.5446 & 0.5011 & $\begin{array}{l}(0.5014, \\
0.6291)\end{array}$ \\
\hline 200 & 0.5097 & 0.4612 & 0.5724 & 0.6128 & 0.6272 & 0.5345 & 0.5118 & 0.5529 & 0.5322 & 0.5579 & 0.5513 & 0.5289 & $\begin{array}{c}(0.4837, \\
0.6094)\end{array}$ \\
\hline 240 & 0.4753 & 0.5412 & 0.6479 & 0.5686 & 0.5948 & 0.5924 & 0.5439 & 0.4881 & 0.4590 & 0.5665 & 0.5625 & 0.5396 & $\begin{array}{c}(0.4857, \\
0.6111)\end{array}$ \\
\hline
\end{tabular}

Table 6. The test results for the overall calendar effect using Method 2

\begin{tabular}{|c|c|c|c|c|c|c|c|c|c|c|c|c|c|}
\hline \multicolumn{14}{|c|}{ HSI } \\
\hline $\begin{array}{l}\text { Moving- } \\
\text { window } \\
\text { size }\end{array}$ & Jan & Feb & Mar & Apr & May & Jun & Jul & Aug & Sep & Oct & Nov & Dec & $\begin{array}{l}95 \% \text { con- } \\
\text { fidence } \\
\text { interval }\end{array}$ \\
\hline 40 & 0.5419 & 0.4800 & 0.4190 & 0.5022 & 0.6099 & 0.4788 & 0.5675 & 0.6523 & 0.5809 & 0.5451 & 0.6250 & 0.6039 & $\begin{array}{c}(0.4887 \\
0.6135)\end{array}$ \\
\hline 80 & 0.6946 & 0.6024 & 0.4881 & 0.4624 & 0.5216 & 0.4610 & 0.5032 & 0.5335 & 0.6297 & 0.5837 & 0.5960 & 0.5974 & $\begin{array}{c}(0.4937 \\
0.6183)\end{array}$ \\
\hline 120 & 0.6194 & 0.5553 & 0.6069 & 0.5819 & 0.5000 & 0.4588 & 0.5289 & 0.4881 & 0.5987 & 0.6137 & 0.6629 & 0.6510 & $\begin{array}{l}(0.5101 \\
0.6344)\end{array}$ \\
\hline 160 & 0.6667 & 0.6471 & 0.5702 & 0.6637 & 0.5927 & 0.5457 & 0.5182 & 0.5810 & 0.5255 & 0.5601 & 0.6228 & 0.6146 & $\begin{array}{c}(0.5300 \\
0.6539)\end{array}$ \\
\hline 200 & 0.6258 & 0.6141 & 0.6242 & 0.6504 & 0.6099 & 0.5768 & 0.6381 & 0.6328 & 0.6497 & 0.5880 & 0.5826 & 0.6124 & $\begin{array}{c}(0.5557, \\
0.6787)\end{array}$ \\
\hline 240 & 0.5462 & 0.5694 & 0.5810 & 0.6062 & 0.6358 & 0.6370 & 0.5653 & 0.6566 & 0.6630 & 0.6373 & 0.5938 & 0.6103 & $\begin{array}{l}(0.5469 \\
0.6702)\end{array}$ \\
\hline \multicolumn{14}{|c|}{ NKY } \\
\hline $\begin{array}{l}\text { Moving- } \\
\text { window } \\
\text { size }\end{array}$ & Jan & Feb & Mar & Apr & May & Jun & Jul & Aug & Sep & Oct & Nov & Dec & $\begin{array}{l}95 \% \text { con- } \\
\text { fidence } \\
\text { interval }\end{array}$ \\
\hline 40 & 0.5548 & 0.5435 & 0.5400 & 0.5907 & 0.5603 & 0.4788 & 0.5396 & 0.4514 & 0.3526 & 0.4249 & 0.5156 & 0.5289 & $\begin{array}{l}(0.4440 \\
0.5695)\end{array}$ \\
\hline 80 & 0.5674 & 0.5341 & 0.6501 & 0.5885 & 0.5323 & 0.5479 & 0.5482 & 0.3542 & 0.3481 & 0.3519 & 0.4107 & 0.4561 & $\begin{array}{c}(0.4286 \\
0.5528)\end{array}$ \\
\hline 120 & 0.5032 & 0.4894 & 0.6069 & 0.6327 & 0.5366 & 0.5234 & 0.5782 & 0.4536 & 0.3481 & 0.2918 & 0.3973 & 0.4561 & $\begin{array}{c}(0.4228, \\
0.5469)\end{array}$ \\
\hline 160 & 0.5011 & 0.4847 & 0.5378 & 0.6084 & 0.5108 & 0.5679 & 0.5118 & 0.4536 & 0.4590 & 0.4185 & 0.3259 & 0.3940 & $\begin{array}{c}(0.4184 \\
0.5436)\end{array}$ \\
\hline 200 & 0.3957 & 0.4376 & 0.5637 & 0.4425 & 0.4547 & 0.5078 & 0.5824 & 0.5529 & 0.4923 & 0.4227 & 0.4397 & 0.4283 & $\begin{array}{c}(0.4142, \\
0.5398)\end{array}$ \\
\hline 240 & 0.5118 & 0.4729 & 0.4320 & 0.4580 & 0.4440 & 0.4120 & 0.4690 & 0.4730 & 0.4967 & 0.5150 & 0.5134 & 0.4839 & $\begin{array}{c}(0.4104 \\
0.5367)\end{array}$ \\
\hline
\end{tabular}


Continue of Table 6

\begin{tabular}{|c|c|c|c|c|c|c|c|c|c|c|c|c|c|}
\hline \multicolumn{14}{|c|}{ SPX } \\
\hline $\begin{array}{c}\text { Moving- } \\
\text { window } \\
\text { size }\end{array}$ & Jan & Feb & Mar & Apr & May & Jun & Jul & Aug & Sep & Oct & Nov & Dec & $\begin{array}{l}95 \% \text { con- } \\
\text { fidence } \\
\text { interval }\end{array}$ \\
\hline 40 & 0.6882 & 0.5671 & 0.6285 & 0.6726 & 0.7069 & 0.5301 & 0.5846 & 0.4687 & 0.5233 & 0.5343 & 0.7254 & 0.7559 & $\begin{array}{l}(0.5553, \\
0.6764)\end{array}$ \\
\hline 80 & 0.7806 & 0.7224 & 0.6760 & 0.6814 & 0.7026 & 0.6258 & 0.6403 & 0.5076 & 0.5721 & 0.5021 & 0.5402 & 0.7859 & $\begin{array}{c}(0.5853, \\
0.7041)\end{array}$ \\
\hline 120 & 0.7011 & 0.7035 & 0.8207 & 0.7522 & 0.6897 & 0.6815 & 0.6938 & 0.6415 & 0.5543 & 0.5429 & 0.6674 & 0.6724 & $\begin{array}{l}(0.6181, \\
0.7352)\end{array}$ \\
\hline 160 & 0.6753 & 0.6706 & 0.6998 & 0.7655 & 0.8362 & 0.7639 & 0.7537 & 0.6782 & 0.6918 & 0.5773 & 0.6451 & 0.6595 & $\begin{array}{c}(0.6441, \\
0.7588)\end{array}$ \\
\hline 200 & 0.6817 & 0.6518 & 0.7149 & 0.7035 & 0.7522 & 0.7951 & 0.7794 & 0.7754 & 0.7384 & 0.6395 & 0.7076 & 0.7195 & $\begin{array}{c}(0.6655, \\
0.7783)\end{array}$ \\
\hline 240 & 0.6753 & 0.6941 & 0.7214 & 0.7035 & 0.7091 & 0.7171 & 0.7816 & 0.7797 & 0.7738 & 0.7318 & 0.7254 & 0.7302 & $\begin{array}{l}(0.6727, \\
0.7850)\end{array}$ \\
\hline \multicolumn{14}{|c|}{ UKX } \\
\hline $\begin{array}{l}\text { Moving- } \\
\text { window } \\
\text { size }\end{array}$ & Jan & Feb & Mar & Apr & May & Jun & Jul & Aug & Sep & Oct & Nov & Dec & $\begin{array}{l}95 \% \text { con- } \\
\text { fidence } \\
\text { interval }\end{array}$ \\
\hline 40 & 0.6882 & 0.6188 & 0.6112 & 0.6350 & 0.6724 & 0.4209 & 0.4818 & 0.4816 & 0.5543 & 0.5665 & 0.6897 & 0.6338 & $\begin{array}{l}(0.5263, \\
0.6492)\end{array}$ \\
\hline 80 & 0.7527 & 0.7012 & 0.6091 & 0.7013 & 0.6810 & 0.5145 & 0.4882 & 0.3974 & 0.5078 & 0.5215 & 0.5558 & 0.6874 & $\begin{array}{l}(0.5319, \\
0.6535)\end{array}$ \\
\hline 120 & 0.6301 & 0.6518 & 0.7775 & 0.6903 & 0.6681 & 0.5813 & 0.5696 & 0.4579 & 0.4946 & 0.5343 & 0.5737 & 0.5739 & $\begin{array}{l}(0.5389, \\
0.6611)\end{array}$ \\
\hline 160 & 0.5763 & 0.5694 & 0.6544 & 0.7323 & 0.7543 & 0.6414 & 0.6338 & 0.5810 & 0.6098 & 0.5429 & 0.5402 & 0.5525 & $\begin{array}{l}(0.5549, \\
0.6769)\end{array}$ \\
\hline 200 & 0.5806 & 0.5506 & 0.6523 & 0.6305 & 0.6573 & 0.6904 & 0.7495 & 0.6393 & 0.6918 & 0.6030 & 0.6228 & 0.5760 & $\begin{array}{c}(0.5769, \\
0.6979)\end{array}$ \\
\hline 240 & 0.6366 & 0.6118 & 0.5961 & 0.5885 & 0.6121 & 0.6080 & 0.6681 & 0.6782 & 0.6984 & 0.6438 & 0.6786 & 0.6617 & $\begin{array}{l}(0.5797, \\
0.7010)\end{array}$ \\
\hline \multicolumn{14}{|c|}{ CAC } \\
\hline $\begin{array}{c}\text { Moving- } \\
\text { window } \\
\text { size }\end{array}$ & Jan & Feb & Mar & Apr & May & Jun & Jul & Aug & Sep & Oct & Nov & Dec & $\begin{array}{c}95 \% \text { con- } \\
\text { fidence } \\
\text { interval }\end{array}$ \\
\hline 40 & 0.6731 & 0.6588 & 0.5832 & 0.6836 & 0.6681 & 0.5301 & 0.4240 & 0.4536 & 0.5854 & 0.5901 & 0.6183 & 0.6274 & $\begin{array}{l}(0.5293, \\
0.6521)\end{array}$ \\
\hline 80 & 0.7355 & 0.6753 & 0.7019 & 0.7080 & 0.6616 & 0.5880 & 0.4989 & 0.4147 & 0.5122 & 0.5215 & 0.5313 & 0.6017 & $\begin{array}{c}(0.5345, \\
0.6564)\end{array}$ \\
\hline 120 & 0.5333 & 0.6824 & 0.7603 & 0.7323 & 0.6616 & 0.5679 & 0.5782 & 0.5119 & 0.5078 & 0.4828 & 0.5469 & 0.5396 & $\begin{array}{c}(0.5302, \\
0.6527)\end{array}$ \\
\hline 160 & 0.5527 & 0.6094 & 0.6458 & 0.7124 & 0.7112 & 0.5857 & 0.6338 & 0.6026 & 0.6585 & 0.4979 & 0.4978 & 0.5332 & $\begin{array}{c}(0.5420, \\
0.6646)\end{array}$ \\
\hline 200 & 0.5763 & 0.6141 & 0.6156 & 0.6173 & 0.6358 & 0.6347 & 0.6531 & 0.6069 & 0.7029 & 0.6824 & 0.6652 & 0.5910 & $\begin{array}{c}(0.5720, \\
0.6937)\end{array}$ \\
\hline 240 & 0.6452 & 0.6565 & 0.6242 & 0.6659 & 0.6530 & 0.6347 & 0.6188 & 0.6479 & 0.6851 & 0.6416 & 0.6875 & 0.6660 & $\begin{array}{c}(0.5917, \\
0.7123)\end{array}$ \\
\hline \multicolumn{14}{|c|}{ DAX } \\
\hline $\begin{array}{c}\text { Moving- } \\
\text { window } \\
\text { size }\end{array}$ & Jan & Feb & Mar & Apr & May & Jun & Jul & Aug & Sep & Oct & Nov & Dec & $\begin{array}{c}95 \% \text { con- } \\
\text { fidence } \\
\text { interval } \\
\end{array}$ \\
\hline 40 & 0.7634 & 0.6706 & 0.5853 & 0.6637 & 0.6659 & 0.5435 & 0.4989 & 0.4579 & 0.5055 & 0.5472 & 0.6585 & 0.7687 & $\begin{array}{c}(0.5494, \\
0.6703) \\
\end{array}$ \\
\hline 80 & 0.7570 & 0.7388 & 0.6890 & 0.6925 & 0.6832 & 0.5991 & 0.5717 & 0.4428 & 0.5388 & 0.5107 & 0.5469 & 0.6874 & $\begin{array}{c}(0.5607, \\
0.6812)\end{array}$ \\
\hline 120 & 0.5806 & 0.6518 & 0.7581 & 0.7611 & 0.7371 & 0.6927 & 0.6852 & 0.5724 & 0.5632 & 0.5322 & 0.5982 & 0.6017 & $\begin{array}{c}(0.5845, \\
0.7042) \\
\end{array}$ \\
\hline 160 & 0.6108 & 0.6165 & 0.6458 & 0.7146 & 0.7909 & 0.7283 & 0.7430 & 0.6587 & 0.6585 & 0.5751 & 0.6317 & 0.6595 & $\begin{array}{c}(0.6106, \\
0.7288)\end{array}$ \\
\hline 200 & 0.6409 & 0.6165 & 0.6220 & 0.6173 & 0.6466 & 0.7038 & 0.7816 & 0.7257 & 0.7228 & 0.6116 & 0.6496 & 0.6745 & $\begin{array}{l}(0.6088, \\
0.7273)\end{array}$ \\
\hline 240 & 0.6968 & 0.6706 & 0.6220 & 0.6173 & 0.6185 & 0.6214 & 0.6488 & 0.7149 & 0.7738 & 0.7189 & 0.7478 & 0.7366 & $\begin{array}{l}(0.6237, \\
0.7409)\end{array}$ \\
\hline
\end{tabular}


Continue of Table 6

\begin{tabular}{|c|c|c|c|c|c|c|c|c|c|c|c|c|c|}
\hline \multicolumn{14}{|c|}{ ELHK } \\
\hline $\begin{array}{l}\text { Moving- } \\
\text { window } \\
\text { size }\end{array}$ & Jan & Feb & Mar & Apr & May & Jun & Jul & Aug & Sep & Oct & Nov & Dec & $\begin{array}{l}95 \% \text { con- } \\
\text { fidence } \\
\text { interval }\end{array}$ \\
\hline 40 & 0.6280 & 0.4424 & 0.4492 & 0.4358 & 0.5841 & 0.4566 & 0.5439 & 0.6220 & 0.6009 & 0.5107 & 0.5067 & 0.5375 & $\begin{array}{l}(0.4647, \\
0.5900)\end{array}$ \\
\hline 80 & 0.6237 & 0.5976 & 0.5092 & 0.4956 & 0.4978 & 0.3987 & 0.4411 & 0.5162 & 0.6208 & 0.6438 & 0.5670 & 0.5289 & $\begin{array}{c}(0.4740, \\
0.5990)\end{array}$ \\
\hline 120 & 0.6473 & 0.5765 & 0.5659 & 0.6062 & 0.5754 & 0.5100 & 0.4518 & 0.4968 & 0.6075 & 0.5494 & 0.6563 & 0.6574 & $\begin{array}{l}(0.5127, \\
0.6369)\end{array}$ \\
\hline 160 & 0.6602 & 0.6494 & 0.5637 & 0.5133 & 0.5733 & 0.5122 & 0.5225 & 0.5745 & 0.5942 & 0.5944 & 0.5938 & 0.6274 & $\begin{array}{l}(0.5192, \\
0.6436)\end{array}$ \\
\hline 200 & 0.5914 & 0.6141 & 0.6371 & 0.6128 & 0.5819 & 0.4744 & 0.5203 & 0.5594 & 0.6231 & 0.6137 & 0.6004 & 0.5974 & $\begin{array}{l}(0.5233, \\
0.6476)\end{array}$ \\
\hline 240 & 0.5806 & 0.5953 & 0.5594 & 0.6460 & 0.6272 & 0.5768 & 0.5632 & 0.6544 & 0.6541 & 0.6416 & 0.5938 & 0.6103 & $\begin{array}{l}(0.5469, \\
0.6703)\end{array}$ \\
\hline \multicolumn{14}{|c|}{ ELJP } \\
\hline $\begin{array}{l}\text { Moving- } \\
\text { window } \\
\text { size }\end{array}$ & Jan & Feb & Mar & Apr & May & Jun & Jul & Aug & Sep & Oct & Nov & Dec & $\begin{array}{l}95 \% \text { con- } \\
\text { fidence } \\
\text { interval }\end{array}$ \\
\hline 40 & 0.4796 & 0.5271 & 0.6264 & 0.6858 & 0.5603 & 0.4187 & 0.5246 & 0.3996 & 0.3947 & 0.5644 & 0.5201 & 0.3854 & $\begin{array}{l}(0.4449, \\
0.5694)\end{array}$ \\
\hline 80 & 0.4710 & 0.4376 & 0.5270 & 0.6239 & 0.6810 & 0.5991 & 0.4561 & 0.3542 & 0.3614 & 0.3755 & 0.4397 & 0.4347 & $\begin{array}{l}(0.4182, \\
0.5421)\end{array}$ \\
\hline 120 & 0.4194 & 0.4306 & 0.5032 & 0.5597 & 0.5733 & 0.5835 & 0.6445 & 0.5724 & 0.3836 & 0.3970 & 0.4107 & 0.4026 & $\begin{array}{l}(0.4282, \\
0.5528)\end{array}$ \\
\hline 160 & 0.4516 & 0.3788 & 0.4795 & 0.5575 & 0.5474 & 0.5078 & 0.5824 & 0.6371 & 0.5787 & 0.5536 & 0.3683 & 0.3533 & $\begin{array}{l}(0.4382, \\
0.5609)\end{array}$ \\
\hline 200 & 0.4237 & 0.4094 & 0.4816 & 0.4270 & 0.5474 & 0.5479 & 0.5546 & 0.5961 & 0.5255 & 0.5858 & 0.6094 & 0.4754 & $\begin{array}{l}(0.4531, \\
0.5786)\end{array}$ \\
\hline 240 & 0.5204 & 0.5082 & 0.4471 & 0.4314 & 0.4332 & 0.4499 & 0.5910 & 0.5702 & 0.4812 & 0.5665 & 0.5089 & 0.5032 & $\begin{array}{l}(0.4383, \\
0.5643)\end{array}$ \\
\hline \multicolumn{14}{|c|}{ UNUS } \\
\hline $\begin{array}{l}\text { Moving- } \\
\text { window } \\
\text { size }\end{array}$ & Jan & Feb & Mar & Apr & May & Jun & Jul & Aug & Sep & Oct & Nov & Dec & $\begin{array}{l}95 \% \text { con- } \\
\text { fidence } \\
\text { interval }\end{array}$ \\
\hline 40 & 0.7484 & 0.6188 & 0.6004 & 0.5774 & 0.6272 & 0.5457 & 0.5632 & 0.6220 & 0.5166 & 0.4571 & 0.4955 & 0.5953 & $\begin{array}{l}(0.5190, \\
0.6427)\end{array}$ \\
\hline 80 & 0.7011 & 0.6682 & 0.7192 & 0.7279 & 0.7069 & 0.6949 & 0.6188 & 0.5896 & 0.5676 & 0.5172 & 0.4040 & 0.5332 & $\begin{array}{l}(0.5604, \\
0.6809)\end{array}$ \\
\hline 120 & 0.5935 & 0.6824 & 0.7473 & 0.8119 & 0.7780 & 0.7884 & 0.7002 & 0.6026 & 0.6208 & 0.5236 & 0.5402 & 0.5418 & $\begin{array}{l}(0.6018, \\
0.7190)\end{array}$ \\
\hline 160 & 0.6043 & 0.5953 & 0.6933 & 0.7611 & 0.8147 & 0.7996 & 0.7002 & 0.6307 & 0.6164 & 0.5773 & 0.5313 & 0.5375 & $\begin{array}{l}(0.5963, \\
0.7143)\end{array}$ \\
\hline 200 & 0.6215 & 0.6141 & 0.6847 & 0.7257 & 0.7909 & 0.8018 & 0.7816 & 0.7235 & 0.6829 & 0.5815 & 0.5826 & 0.6039 & $\begin{array}{l}(0.6251, \\
0.7413)\end{array}$ \\
\hline 240 & 0.6280 & 0.6635 & 0.6544 & 0.6770 & 0.7543 & 0.7105 & 0.7816 & 0.6933 & 0.6851 & 0.6824 & 0.6071 & 0.5803 & $\begin{array}{l}(0.6178, \\
0.7355)\end{array}$ \\
\hline \multicolumn{14}{|c|}{ ELUK } \\
\hline $\begin{array}{l}\text { Moving- } \\
\text { window } \\
\text { size }\end{array}$ & Jan & Feb & Mar & Apr & May & Jun & Jul & Aug & Sep & Oct & Nov & Dec & $\begin{array}{c}95 \% \text { con- } \\
\text { fidence } \\
\text { interval }\end{array}$ \\
\hline 40 & 0.6215 & 0.7318 & 0.6091 & 0.5907 & 0.6724 & 0.5033 & 0.4304 & 0.6026 & 0.6098 & 0.4528 & 0.5156 & 0.4261 & $\begin{array}{l}(0.5009, \\
0.6243)\end{array}$ \\
\hline 80 & 0.6022 & 0.5976 & 0.6566 & 0.6704 & 0.7198 & 0.6258 & 0.6231 & 0.5896 & 0.4922 & 0.5987 & 0.5580 & 0.4968 & $\begin{array}{l}(0.5413, \\
0.6642)\end{array}$ \\
\hline 120 & 0.5806 & 0.5224 & 0.6156 & 0.6217 & 0.6875 & 0.7327 & 0.6231 & 0.5508 & 0.5676 & 0.5515 & 0.5558 & 0.6338 & $\begin{array}{l}(0.5425, \\
0.6655)\end{array}$ \\
\hline 160 & 0.5957 & 0.5765 & 0.6026 & 0.6062 & 0.6559 & 0.6915 & 0.7002 & 0.6609 & 0.6098 & 0.5815 & 0.5536 & 0.5182 & $\begin{array}{c}(0.5516, \\
0.6743) \\
\end{array}$ \\
\hline 200 & 0.6129 & 0.6141 & 0.6069 & 0.6615 & 0.6444 & 0.6771 & 0.6231 & 0.6609 & 0.6386 & 0.5815 & 0.6138 & 0.5803 & $\begin{array}{l}(0.5649, \\
0.6873) \\
\end{array}$ \\
\hline 240 & 0.6280 & 0.6282 & 0.6285 & 0.6217 & 0.6681 & 0.6548 & 0.5610 & 0.6004 & 0.5854 & 0.5451 & 0.5938 & 0.5717 & $\begin{array}{l}(0.5452, \\
0.6686)\end{array}$ \\
\hline
\end{tabular}


End of Table 6

\begin{tabular}{|c|c|c|c|c|c|c|c|c|c|c|c|c|c|}
\hline \multicolumn{14}{|c|}{ EPFR } \\
\hline $\begin{array}{l}\text { Moving- } \\
\text { window } \\
\text { size }\end{array}$ & Jan & Feb & Mar & Apr & May & Jun & Jul & Aug & Sep & Oct & Nov & Dec & $\begin{array}{c}95 \% \text { con- } \\
\text { fidence } \\
\text { interval } \\
\end{array}$ \\
\hline 40 & 0.6538 & 0.6659 & 0.7538 & 0.7080 & 0.6013 & 0.4967 & 0.4540 & 0.5400 & 0.5521 & 0.5451 & 0.5603 & 0.5075 & $\begin{array}{l}(0.5245, \\
0.6474)\end{array}$ \\
\hline 80 & 0.6817 & 0.6400 & 0.6998 & 0.8363 & 0.7909 & 0.6013 & 0.4989 & 0.5205 & 0.5033 & 0.5429 & 0.5179 & 0.5546 & $\begin{array}{l}(0.5555, \\
0.6755)\end{array}$ \\
\hline 120 & 0.6538 & 0.6824 & 0.8078 & 0.7699 & 0.7866 & 0.7506 & 0.6788 & 0.6242 & 0.5322 & 0.4313 & 0.4732 & 0.5803 & $\begin{array}{l}(0.5888, \\
0.7061)\end{array}$ \\
\hline 160 & 0.5484 & 0.6729 & 0.6868 & 0.7566 & 0.7888 & 0.7194 & 0.7323 & 0.7019 & 0.6475 & 0.5815 & 0.5737 & 0.5289 & $\begin{array}{l}(0.6023, \\
0.7203)\end{array}$ \\
\hline 200 & 0.6323 & 0.5976 & 0.6458 & 0.6792 & 0.7198 & 0.7661 & 0.7880 & 0.7538 & 0.7472 & 0.7039 & 0.6540 & 0.6317 & $\begin{array}{l}(0.6359, \\
0.7517)\end{array}$ \\
\hline 240 & 0.7312 & 0.6847 & 0.7019 & 0.6527 & 0.7478 & 0.7506 & 0.7923 & 0.7991 & 0.8204 & 0.6996 & 0.7545 & 0.7216 & $\begin{array}{c}(0.6830, \\
0.7937)\end{array}$ \\
\hline \multicolumn{14}{|c|}{ EPGR } \\
\hline $\begin{array}{c}\text { Moving- } \\
\text { window } \\
\text { size }\end{array}$ & Jan & Feb & Mar & Apr & May & Jun & Jul & Aug & Sep & Oct & Nov & Dec & $\begin{array}{l}95 \% \text { con- } \\
\text { fidence } \\
\text { interval } \\
\end{array}$ \\
\hline 40 & 0.4925 & 0.5953 & 0.5508 & 0.5066 & 0.6293 & 0.5323 & 0.4133 & 0.5248 & 0.5654 & 0.4120 & 0.5246 & 0.4197 & $\begin{array}{l}(0.4502, \\
0.5757)\end{array}$ \\
\hline 80 & 0.4839 & 0.4306 & 0.6415 & 0.6460 & 0.6659 & 0.5768 & 0.4797 & 0.5097 & 0.4324 & 0.4742 & 0.5089 & 0.4497 & $\begin{array}{c}(0.4630, \\
0.5878)\end{array}$ \\
\hline 120 & 0.5075 & 0.4706 & 0.5940 & 0.5642 & 0.7047 & 0.6281 & 0.5182 & 0.5400 & 0.4967 & 0.5150 & 0.5000 & 0.5546 & $\begin{array}{l}(0.4875, \\
0.6125)\end{array}$ \\
\hline 160 & 0.4968 & 0.5529 & 0.6242 & 0.5664 & 0.6272 & 0.6147 & 0.6017 & 0.5659 & 0.5122 & 0.5794 & 0.5379 & 0.5032 & $\begin{array}{l}(0.5028, \\
0.6279)\end{array}$ \\
\hline 200 & 0.5054 & 0.4659 & 0.5637 & 0.6128 & 0.6315 & 0.5412 & 0.5075 & 0.5529 & 0.5322 & 0.5622 & 0.5402 & 0.5314 & $\begin{array}{l}(0.4832, \\
0.6088)\end{array}$ \\
\hline 240 & 0.4688 & 0.5341 & 0.6458 & 0.5686 & 0.5970 & 0.5991 & 0.5439 & 0.4946 & 0.4590 & 0.5622 & 0.5625 & 0.5439 & $\begin{array}{l}(0.4857, \\
0.6110)\end{array}$ \\
\hline
\end{tabular}

Table 7. The test results for the overall calendar effect using Method 3

\begin{tabular}{|c|c|c|c|c|c|c|c|c|c|c|c|c|c|}
\hline \multicolumn{14}{|c|}{ HSI } \\
\hline $\begin{array}{l}\text { Moving- } \\
\text { window } \\
\text { size }\end{array}$ & Jan & Feb & Mar & Apr & May & Jun & Jul & Aug & Sep & Oct & Nov & Dec & $\begin{array}{l}95 \% \text { con- } \\
\text { fidence } \\
\text { interval }\end{array}$ \\
\hline 40 & 0.5441 & 0.4682 & 0.4104 & 0.5000 & 0.5991 & 0.4989 & 0.5601 & 0.6501 & 0.6009 & 0.5494 & 0.6004 & 0.6381 & $\begin{array}{c}(0.4900 \\
0.6148)\end{array}$ \\
\hline 80 & 0.6925 & 0.6071 & 0.4924 & 0.4602 & 0.5259 & 0.4744 & 0.4989 & 0.5140 & 0.6386 & 0.5815 & 0.5960 & 0.5974 & $\begin{array}{c}(0.4941, \\
0.6187)\end{array}$ \\
\hline 120 & 0.6172 & 0.5553 & 0.6048 & 0.5730 & 0.5022 & 0.4477 & 0.5180 & 0.4881 & 0.5831 & 0.6159 & 0.6607 & 0.6510 & $\begin{array}{l}0.5060, \\
0.6305)\end{array}$ \\
\hline 160 & 0.6602 & 0.6376 & 0.5832 & 0.6571 & 0.5948 & 0.5546 & 0.5118 & 0.5810 & 0.5322 & 0.5451 & 0.6205 & 0.6103 & $\begin{array}{l}(0.5283, \\
0.6523)\end{array}$ \\
\hline 200 & 0.6258 & 0.6188 & 0.6264 & 0.6482 & 0.6142 & 0.5880 & 0.6510 & 0.6307 & 0.6452 & 0.6009 & 0.5893 & 0.6103 & $\begin{array}{l}(0.5594, \\
0.6822)\end{array}$ \\
\hline 240 & 0.5505 & 0.5671 & 0.7502 & 0.6040 & 0.6358 & 0.6370 & 0.5696 & 0.6544 & 0.6630 & 0.6459 & 0.6004 & 0.6103 & $\begin{array}{l}(0.5475, \\
0.6708) \\
\end{array}$ \\
\hline \multicolumn{14}{|c|}{$\mathrm{NKY}$} \\
\hline $\begin{array}{l}\text { Moving- } \\
\text { window } \\
\text { size }\end{array}$ & Jan & Feb & Mar & Apr & May & Jun & Jul & Aug & Sep & Oct & Nov & Dec & $\begin{array}{l}95 \% \text { con- } \\
\text { fidence } \\
\text { interval }\end{array}$ \\
\hline 40 & 0.5462 & 0.5506 & 0.5378 & 0.5885 & 0.5603 & 0.4811 & 0.5546 & 0.4536 & 0.3437 & 0.4335 & 0.5268 & 0.5161 & $\begin{array}{l}(0.4449, \\
0.5704)\end{array}$ \\
\hline 80 & 0.5656 & 0.5412 & 0.6458 & 0.5996 & 0.5453 & 0.5457 & 0.5675 & 0.3629 & 0.3326 & 0.3541 & 0.3951 & 0.4625 & $\begin{array}{l}(0.4311, \\
0.5551)\end{array}$ \\
\hline 120 & 0.5032 & 0.4847 & 0.6004 & 0.6350 & 0.5431 & 0.5301 & 0.5803 & 0.4536 & 0.3614 & 0.2983 & 0.3906 & 0.4668 & $\begin{array}{l}(0.4253, \\
0.5495)\end{array}$ \\
\hline 160 & 0.5032 & 0.4871 & 0.5335 & 0.6106 & 0.5129 & 0.5523 & 0.5075 & 0.4536 & 0.4678 & 0.4120 & 0.3304 & 0.3790 & $\begin{array}{l}0.4164, \\
0.5416)\end{array}$ \\
\hline 200 & 0.3957 & 0.4471 & 0.5680 & 0.4469 & 0.4591 & 0.5056 & 0.5696 & 0.5421 & 0.4967 & 0.4270 & 0.4464 & 0.4390 & $\begin{array}{l}0.4159, \\
0.5417)\end{array}$ \\
\hline 240 & 0.5118 & 0.4894 & 0.4255 & 0.4580 & 0.4418 & 0.4098 & 0.4668 & 0.4687 & 0.5033 & 0.5193 & 0.5156 & 0.4839 & $\begin{array}{l}(0.4113, \\
0.5376)\end{array}$ \\
\hline
\end{tabular}


Continue of Table 7

\begin{tabular}{|c|c|c|c|c|c|c|c|c|c|c|c|c|c|}
\hline \multicolumn{14}{|c|}{ SPX } \\
\hline $\begin{array}{l}\text { Moving- } \\
\text { window } \\
\text { size }\end{array}$ & Jan & Feb & Mar & Apr & May & Jun & Jul & Aug & Sep & Oct & Nov & Dec & $\begin{array}{c}95 \% \text { con- } \\
\text { fidence } \\
\text { interval }\end{array}$ \\
\hline 40 & 0.7032 & 0.5529 & 0.6199 & 0.6814 & 0.7263 & 0.5367 & 0.5803 & 0.4795 & 0.5299 & 0.5279 & 0.7031 & 0.7049 & $\begin{array}{l}(0.5551, \\
0.6763)\end{array}$ \\
\hline 80 & 0.7828 & 0.7388 & 0.6868 & 0.6836 & 0.7134 & 0.6236 & 0.6445 & 0.5076 & 0.5721 & 0.5043 & 0.5402 & 0.8009 & $\begin{array}{l}(0.5907, \\
0.7089)\end{array}$ \\
\hline 120 & 0.6989 & 0.7035 & 0.8143 & 0.7677 & 0.6961 & 0.6993 & 0.7045 & 0.6393 & 0.5543 & 0.5429 & 0.6652 & 0.6724 & $\begin{array}{l}(0.6214, \\
0.7381) \\
\end{array}$ \\
\hline 160 & 0.6667 & 0.6729 & 0.7019 & 0.7611 & 0.8384 & 0.7595 & 0.7537 & 0.6911 & 0.6940 & 0.5880 & 0.6272 & 0.6574 & $\begin{array}{l}(0.6437, \\
0.7585)\end{array}$ \\
\hline 200 & 0.6903 & 0.6588 & 0.7171 & 0.7102 & 0.7522 & 0.7996 & 0.7752 & 0.7819 & 0.7339 & 0.6416 & 0.7165 & 0.7152 & $\begin{array}{c}(0.6683, \\
0.7809) \\
\end{array}$ \\
\hline 240 & 0.6796 & 0.6918 & 0.7106 & 0.7124 & 0.7134 & 0.7060 & 0.7773 & 0.7840 & 0.7627 & 0.7382 & 0.7478 & 0.7238 & $\begin{array}{c}(0.6731, \\
0.7853) \\
\end{array}$ \\
\hline \multicolumn{14}{|c|}{ UKX } \\
\hline $\begin{array}{l}\text { Moving- } \\
\text { window } \\
\text { size }\end{array}$ & Jan & Feb & Mar & Apr & May & Jun & Jul & Aug & Sep & Oct & Nov & Dec & $\begin{array}{c}95 \% \text { con- } \\
\text { fidence } \\
\text { interval } \\
\end{array}$ \\
\hline 40 & 0.6989 & 0.6212 & 0.5983 & 0.6726 & 0.6767 & 0.4410 & 0.4839 & 0.4968 & 0.5543 & 0.5451 & 0.6875 & 0.6531 & $\begin{array}{c}(0.5327, \\
0.6553) \\
\end{array}$ \\
\hline 80 & 0.7570 & 0.7082 & 0.6177 & 0.7080 & 0.6681 & 0.5345 & 0.4882 & 0.3909 & 0.4922 & 0.5322 & 0.5469 & 0.6959 & $\begin{array}{l}(0.5339 \\
0.6552)\end{array}$ \\
\hline 120 & 0.6301 & 0.6494 & 0.7840 & 0.7013 & 0.6530 & 0.5991 & 0.5696 & 0.4730 & 0.4878 & 0.5279 & 0.5714 & 0.5739 & $\begin{array}{l}(0.5424, \\
0.6625)\end{array}$ \\
\hline 160 & 0.5634 & 0.5671 & 0.6458 & 0.7323 & 0.7651 & 0.6526 & 0.6445 & 0.5832 & 0.6075 & 0.5451 & 0.5357 & 0.5460 & $\begin{array}{c}(0.5549, \\
0.6768) \\
\end{array}$ \\
\hline 200 & 0.5935 & 0.5529 & 0.6479 & 0.6327 & 0.6509 & 0.6704 & 0.7323 & 0.6415 & 0.6984 & 0.6030 & 0.6295 & 0.5717 & $\begin{array}{c}(0.5751, \\
0.6964) \\
\end{array}$ \\
\hline 240 & 0.6387 & 0.6165 & 0.5847 & 0.5863 & 0.6099 & 0.5991 & 0.6638 & 0.6847 & 0.6962 & 0.6545 & 0.6786 & 0.6617 & $\begin{array}{c}(0.5793, \\
0.7006)\end{array}$ \\
\hline \multicolumn{14}{|c|}{ CAC } \\
\hline $\begin{array}{l}\text { Moving- } \\
\text { window } \\
\text { size }\end{array}$ & Jan & Feb & Mar & Apr & May & Jun & Jul & Aug & Sep & Oct & Nov & Dec & $\begin{array}{c}95 \% \text { con- } \\
\text { fidence } \\
\text { interval }\end{array}$ \\
\hline 40 & 0.6731 & 0.6659 & 0.5832 & 0.6947 & 0.6789 & 0.5590 & 0.4111 & 0.4600 & 0.5632 & 0.6009 & 0.6094 & 0.6403 & $\begin{array}{c}(0.5331, \\
0.6556)\end{array}$ \\
\hline 80 & 0.7376 & 0.6776 & 0.6847 & 0.7279 & 0.6616 & 0.5991 & 0.5139 & 0.4147 & 0.5011 & 0.5150 & 0.5335 & 0.5974 & $\begin{array}{c}(0.5356 \\
0.6574)\end{array}$ \\
\hline 120 & 0.5161 & 0.6965 & 0.7495 & 0.7367 & 0.6638 & 0.5679 & 0.5739 & 0.5119 & 0.5144 & 0.4785 & 0.5536 & 0.5353 & $\begin{array}{l}(0.5295, \\
0.6519)\end{array}$ \\
\hline 160 & 0.5419 & 0.6094 & 0.6415 & 0.7168 & 0.7198 & 0.5913 & 0.6253 & 0.6026 & 0.6608 & 0.5021 & 0.5089 & 0.5289 & $\begin{array}{l}(0.5418, \\
0.6644)\end{array}$ \\
\hline 200 & 0.5828 & 0.6235 & 0.6112 & 0.6173 & 0.6466 & 0.6303 & 0.6617 & 0.6026 & 0.6984 & 0.6888 & 0.6674 & 0.5931 & $\begin{array}{l}(0.5744, \\
0.6960)\end{array}$ \\
\hline 240 & 0.6366 & 0.6588 & 0.6220 & 0.6659 & 0.6552 & 0.6325 & 0.6146 & 0.6523 & 0.6829 & 0.6524 & 0.6964 & 0.6660 & $\begin{array}{l}(0.5925, \\
0.7130)\end{array}$ \\
\hline \multicolumn{14}{|c|}{ DAX } \\
\hline $\begin{array}{l}\text { Moving- } \\
\text { window } \\
\text { size }\end{array}$ & Jan & Feb & Mar & Apr & May & Jun & Jul & Aug & Sep & Oct & Nov & Dec & $\begin{array}{c}95 \% \text { con- } \\
\text { fidence } \\
\text { interval }\end{array}$ \\
\hline 40 & 0.7871 & 0.6706 & 0.5810 & 0.6681 & 0.6681 & 0.5523 & 0.4882 & 0.4579 & 0.5055 & 0.5429 & 0.6563 & 0.7645 & $\begin{array}{l}(0.5513, \\
0.6720)\end{array}$ \\
\hline 80 & 0.7806 & 0.7412 & 0.6890 & 0.6991 & 0.6961 & 0.6080 & 0.5761 & 0.4406 & 0.5277 & 0.5150 & 0.5379 & 0.6788 & $\begin{array}{c}(0.5637, \\
0.6838) \\
\end{array}$ \\
\hline 120 & 0.5849 & 0.6518 & 0.7538 & 0.7611 & 0.7371 & 0.7194 & 0.6767 & 0.5637 & 0.5676 & 0.5279 & 0.6004 & 0.5953 & $\begin{array}{c}(0.5849, \\
0.7045) \\
\end{array}$ \\
\hline 160 & 0.6129 & 0.6165 & 0.6436 & 0.7058 & 0.7888 & 0.7261 & 0.7409 & 0.6587 & 0.6696 & 0.5687 & 0.6250 & 0.6681 & $\begin{array}{c}(0.6099, \\
0.7281) \\
\end{array}$ \\
\hline 200 & 0.6430 & 0.6165 & 0.6220 & 0.6173 & 0.6444 & 0.7016 & 0.7816 & 0.7408 & 0.7472 & 0.6116 & 0.6696 & 0.6767 & $\begin{array}{c}(0.6140, \\
0.7320) \\
\end{array}$ \\
\hline 240 & 0.6989 & 0.6682 & 0.6220 & 0.6173 & 0.6185 & 0.6214 & 0.6467 & 0.7063 & 0.7738 & 0.7210 & 0.7478 & 0.7409 & $\begin{array}{l}(0.6233, \\
0.7405)\end{array}$ \\
\hline
\end{tabular}


Continue of Table 7

\begin{tabular}{|c|c|c|c|c|c|c|c|c|c|c|c|c|c|}
\hline \multicolumn{14}{|c|}{ ELHK } \\
\hline $\begin{array}{c}\text { Moving- } \\
\text { window } \\
\text { size }\end{array}$ & Jan & Feb & Mar & Apr & May & Jun & Jul & Aug & Sep & Oct & Nov & Dec & $\begin{array}{c}95 \% \text { con- } \\
\text { fidence } \\
\text { interval } \\
\end{array}$ \\
\hline 40 & 0.6043 & 0.4565 & 0.4384 & 0.4270 & 0.5819 & 0.4588 & 0.5310 & 0.6199 & 0.6120 & 0.5172 & 0.5045 & 0.5482 & $\begin{array}{c}(0.4630, \\
0.5884) \\
\end{array}$ \\
\hline 80 & 0.6215 & 0.5976 & 0.4989 & 0.4801 & 0.4935 & 0.4076 & 0.4368 & 0.5140 & 0.6075 & 0.6545 & 0.5737 & 0.5375 & $\begin{array}{c}(0.4725, \\
0.5975) \\
\end{array}$ \\
\hline 120 & 0.6473 & 0.5741 & 0.5594 & 0.6040 & 0.5862 & 0.5145 & 0.4647 & 0.4903 & 0.6208 & 0.5536 & 0.6518 & 0.6617 & $\begin{array}{c}(0.5151, \\
0.6393)\end{array}$ \\
\hline 160 & 0.6645 & 0.6541 & 0.5680 & 0.5089 & 0.5711 & 0.5212 & 0.5161 & 0.5724 & 0.6031 & 0.5966 & 0.5915 & 0.6253 & $\begin{array}{l}(0.5203, \\
0.6446)\end{array}$ \\
\hline 200 & 0.5892 & 0.6071 & 0.6436 & 0.6217 & 0.5841 & 0.4900 & 0.5203 & 0.5616 & 0.6142 & 0.6223 & 0.5982 & 0.5974 & $\begin{array}{c}(0.5253, \\
0.6495)\end{array}$ \\
\hline 240 & 0.5785 & 0.5929 & 0.5551 & 0.6416 & 0.6250 & 0.5724 & 0.5632 & 0.6501 & 0.6563 & 0.6438 & 0.5960 & 0.5974 & $\begin{array}{c}(0.5443, \\
0.6678) \\
\end{array}$ \\
\hline \multicolumn{14}{|c|}{ ELJP } \\
\hline $\begin{array}{c}\text { Moving- } \\
\text { window } \\
\text { size }\end{array}$ & Jan & Feb & Mar & Apr & May & Jun & Jul & Aug & Sep & Oct & Nov & Dec & $\begin{array}{l}95 \% \text { con- } \\
\text { fidence } \\
\text { interval }\end{array}$ \\
\hline 40 & 0.4710 & 0.5082 & 0.6285 & 0.6881 & 0.5668 & 0.4187 & 0.5353 & 0.4107 & 0.3814 & 0.5472 & 0.5313 & 0.4004 & $\begin{array}{l}(0.4443, \\
0.5688)\end{array}$ \\
\hline 80 & 0.4753 & 0.4282 & 0.5292 & 0.6150 & 0.6875 & 0.6058 & 0.4711 & 0.3473 & 0.3659 & 0.3670 & 0.4420 & 0.4325 & $\begin{array}{c}(0.4188, \\
0.5426)\end{array}$ \\
\hline 120 & 0.4172 & 0.4282 & 0.5101 & 0.5553 & 0.5884 & 0.5746 & 0.6424 & 0.5918 & 0.3969 & 0.4077 & 0.4174 & 0.4047 & $\begin{array}{c}(0.4320, \\
0.5567)\end{array}$ \\
\hline 160 & 0.4624 & 0.3812 & 0.4773 & 0.5597 & 0.5819 & 0.5033 & 0.5675 & 0.6479 & 0.5698 & 0.5579 & 0.3817 & 0.3448 & $\begin{array}{l}(0.4415, \\
0.5661)\end{array}$ \\
\hline 200 & 0.4258 & 0.4000 & 0.4838 & 0.4336 & 0.5323 & 0.5546 & 0.5567 & 0.5940 & 0.5322 & 0.5987 & 0.6027 & 0.4754 & $\begin{array}{c}0.4537, \\
0.5791)\end{array}$ \\
\hline 240 & 0.5247 & 0.5059 & 0.4471 & 0.4381 & 0.4353 & 0.4454 & 0.5846 & 0.5637 & 0.4656 & 0.5687 & 0.5067 & 0.5032 & $\begin{array}{c}(0.4365, \\
0.5625) \\
\end{array}$ \\
\hline \multicolumn{14}{|c|}{ UNUS } \\
\hline $\begin{array}{l}\text { Moving- } \\
\text { window } \\
\text { size }\end{array}$ & Jan & Feb & Mar & Apr & May & Jun & Jul & Aug & Sep & Oct & Nov & Dec & $\begin{array}{l}95 \% \text { con- } \\
\text { fidence } \\
\text { interval } \\
\end{array}$ \\
\hline 40 & 0.7398 & 0.6282 & 0.6004 & 0.5863 & 0.6185 & 0.5367 & 0.5503 & 0.6393 & 0.5299 & 0.4464 & 0.4955 & 0.5867 & $\begin{array}{l}(0.5181, \\
0.6418)\end{array}$ \\
\hline 80 & 0.6968 & 0.6941 & 0.7171 & 0.7301 & 0.7069 & 0.7105 & 0.6124 & 0.5875 & 0.5698 & 0.5215 & 0.4018 & 0.5161 & $\begin{array}{c}(0.5616, \\
0.6818)\end{array}$ \\
\hline 120 & 0.5935 & 0.6776 & 0.7451 & 0.8142 & 0.7888 & 0.7862 & 0.7045 & 0.6048 & 0.6160 & 0.5107 & 0.5379 & 0.5503 & $\begin{array}{c}(0.6018, \\
0.7190)\end{array}$ \\
\hline 160 & 0.5978 & 0.5882 & 0.6825 & 0.7633 & 0.8103 & 0.7951 & 0.7024 & 0.6307 & 0.6186 & 0.5880 & 0.5357 & 0.5375 & $\begin{array}{c}(0.5953, \\
0.7135) \\
\end{array}$ \\
\hline 200 & 0.6215 & 0.6144 & 0.6782 & 0.7279 & 0.7845 & 0.8018 & 0.7816 & 0.7171 & 0.6785 & 0.5708 & 0.5804 & 0.6060 & $\begin{array}{c}(0.6223, \\
0.7387) \\
\end{array}$ \\
\hline 240 & 0.6258 & 0.6635 & 0.6458 & 0.6748 & 0.7522 & 0.7171 & 0.7816 & 0.6933 & 0.6940 & 0.6738 & 0.6094 & 0.5782 & $\begin{array}{l}0.6170, \\
0.7348) \\
\end{array}$ \\
\hline \multicolumn{14}{|c|}{ ELUK } \\
\hline $\begin{array}{l}\text { Moving- } \\
\text { window } \\
\text { size }\end{array}$ & Jan & Feb & Mar & Apr & May & Jun & Jul & Aug & Sep & Oct & Nov & Dec & $\begin{array}{c}95 \% \text { con- } \\
\text { fidence } \\
\text { interval }\end{array}$ \\
\hline 40 & 0.6172 & 0.7412 & 0.6199 & 0.6018 & 0.6703 & 0.5212 & 0.4240 & 0.5940 & 0.6208 & 0.4592 & 0.5357 & 0.4347 & $\begin{array}{l}(0.5070, \\
0.6302)\end{array}$ \\
\hline 80 & 0.6000 & 0.6000 & 0.6501 & 0.6726 & 0.7155 & 0.6459 & 0.6081 & 0.5961 & 0.4945 & 0.5966 & 0.5670 & 0.4968 & $\begin{array}{c}(0.5422, \\
0.6651) \\
\end{array}$ \\
\hline 120 & 0.5828 & 0.5200 & 0.6134 & 0.5973 & 0.6724 & 0.7416 & 0.6296 & 0.5464 & 0.5654 & 0.5494 & 0.5625 & 0.6317 & $\begin{array}{l}(0.5399, \\
0.6630) \\
\end{array}$ \\
\hline 160 & 0.5957 & 0.5812 & 0.6134 & 0.6128 & 0.6703 & 0.6860 & 0.6981 & 0.6587 & 0.6053 & 0.5880 & 0.5603 & 0.5096 & $\begin{array}{c}(0.5539, \\
0.6764) \\
\end{array}$ \\
\hline 200 & 0.6215 & 0.6141 & 0.6069 & 0.6593 & 0.6466 & 0.6478 & 0.6231 & 0.6652 & 0.6475 & 0.5665 & 0.6161 & 0.5803 & $\begin{array}{l}(0.5655, \\
0.6878) \\
\end{array}$ \\
\hline 240 & 0.6301 & 0.6212 & 0.6328 & 0.6173 & 0.6681 & 0.6592 & 0.5675 & 0.5983 & 0.5920 & 0.5451 & 0.5982 & 0.5675 & $\begin{array}{c}(0.5462, \\
0.6695)\end{array}$ \\
\hline
\end{tabular}


End of Table 7

\begin{tabular}{|c|c|c|c|c|c|c|c|c|c|c|c|c|c|}
\hline \multicolumn{14}{|c|}{ EPFR } \\
\hline $\begin{array}{l}\text { Moving- } \\
\text { window } \\
\text { size }\end{array}$ & Jan & Feb & Mar & Apr & May & Jun & Jul & Aug & Sep & Oct & Nov & Dec & $\begin{array}{l}95 \% \text { con- } \\
\text { fidence } \\
\text { interval } \\
\end{array}$ \\
\hline 40 & 0.6602 & 0.6706 & 0.7689 & 0.7323 & 0.5970 & 0.4967 & 0.4475 & 0.5486 & 0.5521 & 0.5494 & 0.5536 & 0.5225 & $\begin{array}{c}(0.5299 \\
0.6522)\end{array}$ \\
\hline 80 & 0.6774 & 0.6471 & 0.7171 & 0.8473 & 0.8060 & 0.6236 & 0.5011 & 0.5205 & 0.5100 & 0.5429 & 0.5179 & 0.5439 & $\begin{array}{l}(0.5613, \\
0.6807)\end{array}$ \\
\hline 120 & 0.6495 & 0.6753 & 0.8056 & 0.7611 & 0.7823 & 0.7617 & 0.6724 & 0.6242 & 0.5565 & 0.4356 & 0.4665 & 0.5846 & $\begin{array}{l}(0.5891, \\
0.7065)\end{array}$ \\
\hline 160 & 0.5591 & 0.6635 & 0.6847 & 0.7589 & 0.7888 & 0.7216 & 0.7173 & 0.6998 & 0.6608 & 0.5880 & 0.5826 & 0.5332 & $\begin{array}{l}(0.6039, \\
0.7220)\end{array}$ \\
\hline 200 & 0.6366 & 0.5953 & 0.6501 & 0.6792 & 0.7112 & 0.7728 & 0.7923 & 0.7538 & 0.7539 & 0.7210 & 0.6563 & 0.6296 & $\begin{array}{c}(0.6388, \\
0.7543)\end{array}$ \\
\hline 240 & 0.7312 & 0.6776 & 0.7127 & 0.6527 & 0.7414 & 0.7528 & 0.7859 & 0.8056 & 0.8204 & 0.6996 & 0.7388 & 0.7302 & $\begin{array}{l}(0.6824, \\
0.7932)\end{array}$ \\
\hline \multicolumn{14}{|c|}{ EPGR } \\
\hline $\begin{array}{l}\text { Moving- } \\
\text { window } \\
\text { size }\end{array}$ & Jan & Feb & Mar & Apr & May & Jun & Jul & Aug & Sep & Oct & Nov & Dec & $\begin{array}{l}95 \% \text { con- } \\
\text { fidence } \\
\text { interval }\end{array}$ \\
\hline 40 & 0.5054 & 0.5765 & 0.5529 & 0.4956 & 0.6358 & 0.5367 & 0.4133 & 0.5076 & 0.5876 & 0.4227 & 0.5201 & 0.4218 & $\begin{array}{l}(0.4511, \\
0.5766)\end{array}$ \\
\hline 80 & 0.4817 & 0.4353 & 0.6328 & 0.6549 & 0.6466 & 0.5902 & 0.4946 & 0.4968 & 0.4146 & 0.4785 & 0.5022 & 0.4668 & $\begin{array}{l}(0.4626, \\
0.5874)\end{array}$ \\
\hline 120 & 0.4989 & 0.4706 & 0.5940 & 0.5730 & 0.7091 & 0.6370 & 0.5203 & 0.5378 & 0.5033 & 0.5107 & 0.5022 & 0.5525 & $\begin{array}{l}(0.4888, \\
0.6137)\end{array}$ \\
\hline 160 & 0.5140 & 0.5529 & 0.6220 & 0.5730 & 0.6336 & 0.6214 & 0.5996 & 0.5659 & 0.5144 & 0.5794 & 0.5335 & 0.5096 & $\begin{array}{l}(0.5059, \\
0.6309)\end{array}$ \\
\hline 200 & 0.5032 & 0.4706 & 0.5551 & 0.6173 & 0.6422 & 0.5479 & 0.5032 & 0.5378 & 0.5344 & 0.5601 & 0.5357 & 0.5353 & $\begin{array}{l}(0.4828, \\
0.6084)\end{array}$ \\
\hline 240 & 0.4667 & 0.5318 & 0.6415 & 0.5796 & 0.5711 & 0.6080 & 0.5439 & 0.4881 & 0.4545 & 0.5536 & 0.5647 & 0.5503 & $\begin{array}{l}(0.4835, \\
0.6089)\end{array}$ \\
\hline
\end{tabular}

None of the entries in Table 4 are colored, indicating that at 5\% level, no significant calendar effects are found for all the 12 stock indices. However, some entries in Tables 5, 6 and 7 are colored, indicating significant calendar effects at $5 \%$ level. This reveals that additional channels of calendar effects are found by our methods.

In particular, for the 4 western general equity indices (SPX, UKX, CAC and DAX) with moving-window size 80 or smaller, the entries in the column January are green in color, indicating that the January effect is significant. This is natural because the most of the previous studies on January effect work on equity markets of European and North American countries (see Section 1), so the four western general equity indices behave normally. The small moving-window size means that the "delaying effect" (see Hui and Chan, 2016 for detail explanation) is not so significant that the calendar effect is not distorted.

There are several similarities between our results and Hui and Chan (2016)'s results. Firstly, when the movingwindow size increases, there are generally fewer green/ red entries in Tables 5, 6 and 7 and they shift rightwards, showing that the overall calendar effect becomes less prevalent and delays. The reason for this result is explained in Hui and Chan (2016). Secondly, for January, there are more green entries than red entries for the six general equity indices, showing significant January effect. However, the numbers of green and red entries are roughly the same for the six securitized real estate indices, showing no clear signs of the January effect. Furthermore, there are more green entries for smaller moving-window sizes, indicating that the January effect is more significant.

Comparing the results in Tables 5, 6 and 7, we can see that the calendar effects shown by Methods 1, 2 and 3 are slightly different. For example, for HSI index with a moving-window size of 40 , there is a significant positive calendar effect at 5\% level in November for Methods 1 and 2, but not for Method 3. This implies that for both Strategies 1 and 2, we should hold HSI significantly more in November, but this is not the case for Strategy 3. To see the difference in calendar effects shown by the three methods, we compare the number of green and red entries in Tables 5, 6 and 7, find out the differences and list out the differences in the Table 8.

From Table 8, we can see that when one changes from Method 1 to Method 2, the number of green and red entries decreases in general, showing less significant overall calendar effect. This result is expected because for Strategy 1 , one has to buy or sell the stock index immediately as soon as $\hat{\mu}_{i}(n)$ changes sign. However, for Strategy 2, one waits until the sign of $\hat{\mu}_{i}(n)$ remains unchanged for 2 consecutive days before trading the stock index. This creates a "smoothing effect", reducing the number of times of trading the stock index. Therefore, the dummy variable $R_{2 i}$ fluctuates less frequently than $R_{1 i}$ does. This explains why Method 2 shows less significant calendar effect than Method 1 does. However, when one changes from Method 2 to Method 3, the number of green and red entries increases in general, revealing that the overall calendar effect 
Table 8. Change in number of green and red entries between the three methods for the 12 stock indices

\begin{tabular}{|c|c|c|c|}
\hline Index & Method $1 \rightarrow$ Method 2 & Method $2 \rightarrow$ Method 3 & Method $1 \rightarrow$ Method 3 \\
\hline HSI & $\begin{array}{l}\text { No color } \rightarrow \text { green/red: } 0 \\
\text { Green/red } \rightarrow \text { no color: } 0\end{array}$ & $\begin{array}{l}\text { No color } \rightarrow \text { green/red: } 1 \\
\text { Green/red } \rightarrow \text { no color: } 2\end{array}$ & $\begin{array}{l}\text { No color } \rightarrow \text { green/red: } 1 \\
\text { Green/red } \rightarrow \text { no color: } 2\end{array}$ \\
\hline NKY & $\begin{array}{l}\text { No color } \rightarrow \text { green/red: } 1 \\
\text { Green/red } \rightarrow \text { no color: } 2\end{array}$ & $\begin{array}{l}\text { No color } \rightarrow \text { green/red: } 3 \\
\text { Green/red } \rightarrow \text { no color: } 0\end{array}$ & $\begin{array}{l}\text { No color } \rightarrow \text { green/red: } 4 \\
\text { Green/red } \rightarrow \text { no color: } 2\end{array}$ \\
\hline SPX & $\begin{array}{l}\text { No color } \rightarrow \text { green } / \text { red: } 0 \\
\text { Green/red } \rightarrow \text { no color: } 4\end{array}$ & $\begin{array}{l}\text { No color } \rightarrow \text { green/red: } 5 \\
\text { Green/red } \rightarrow \text { no color: } 1\end{array}$ & $\begin{array}{l}\text { No color } \rightarrow \text { green/red: } 2 \\
\text { Green/red } \rightarrow \text { no color: } 2\end{array}$ \\
\hline UKX & $\begin{array}{l}\text { No color } \rightarrow \text { green/red: } 0 \\
\text { Green/red } \rightarrow \text { no color: } 0\end{array}$ & $\begin{array}{l}\text { No color } \rightarrow \text { green/red: } 5 \\
\text { Green/red } \rightarrow \text { no color: } 2\end{array}$ & $\begin{array}{l}\text { No color } \rightarrow \text { green/red: } 5 \\
\text { Green/red } \rightarrow \text { no color: } 2\end{array}$ \\
\hline CAC & $\begin{array}{l}\text { No color } \rightarrow \text { green/red: } 1 \\
\text { Green/red } \rightarrow \text { no color: } 1\end{array}$ & $\begin{array}{l}\text { No color } \rightarrow \text { green/red: } 1 \\
\text { Green/red } \rightarrow \text { no color: } 0\end{array}$ & $\begin{array}{l}\text { No color } \rightarrow \text { green/red: } 2 \\
\text { Green/red } \rightarrow \text { no color: } 1\end{array}$ \\
\hline DAX & $\begin{array}{l}\text { No color } \rightarrow \text { green/red: } 2 \\
\text { Green/red } \rightarrow \text { no color: } 5\end{array}$ & $\begin{array}{l}\text { No color } \rightarrow \text { green/red: } 5 \\
\text { Green/red } \rightarrow \text { no color: } 3\end{array}$ & $\begin{array}{l}\text { No color } \rightarrow \text { green/red: } 4 \\
\text { Green/red } \rightarrow \text { no color: } 5\end{array}$ \\
\hline ELHK & $\begin{array}{l}\text { No color } \rightarrow \text { green/red: } 1 \\
\text { Green/red } \rightarrow \text { no color: } 3\end{array}$ & $\begin{array}{l}\text { No color } \rightarrow \text { green/red: } 4 \\
\text { Green/red } \rightarrow \text { no color: } 1\end{array}$ & $\begin{array}{l}\text { No color } \rightarrow \text { green/red: } 2 \\
\text { Green/red } \rightarrow \text { no color: } 1\end{array}$ \\
\hline ELJP & $\begin{array}{l}\text { No color } \rightarrow \text { green/red: } 1 \\
\text { Green/red } \rightarrow \text { no color: } 6\end{array}$ & $\begin{array}{l}\text { No color } \rightarrow \text { green/red: } 8 \\
\text { Green/red } \rightarrow \text { no color: } 2\end{array}$ & $\begin{array}{l}\text { No color } \rightarrow \text { green/red: } 3 \\
\text { Green/red } \rightarrow \text { no color: } 2\end{array}$ \\
\hline UNUS & $\begin{array}{l}\text { No color } \rightarrow \text { green/red: } 1 \\
\text { Green/red } \rightarrow \text { no color: } 6\end{array}$ & $\begin{array}{l}\text { No color } \rightarrow \text { green/red: } 6 \\
\text { Green/red } \rightarrow \text { no color: } 1\end{array}$ & $\begin{array}{l}\text { No color } \rightarrow \text { green/red: } 2 \\
\text { Green/red } \rightarrow \text { no color: } 2\end{array}$ \\
\hline ELUK & $\begin{array}{l}\text { No color } \rightarrow \text { green/red: } 1 \\
\text { Green/red } \rightarrow \text { no color: } 4\end{array}$ & $\begin{array}{l}\text { No color } \rightarrow \text { green/red: } 0 \\
\text { Green/red } \rightarrow \text { no color: } 1\end{array}$ & $\begin{array}{l}\text { No color } \rightarrow \text { green/red: } 1 \\
\text { Green/red } \rightarrow \text { no color: } 5\end{array}$ \\
\hline EPFR & $\begin{array}{l}\text { No color } \rightarrow \text { green/red: } 2 \\
\text { Green/red } \rightarrow \text { no color: } 1\end{array}$ & $\begin{array}{l}\text { No color } \rightarrow \text { green } / \text { red: } 0 \\
\text { Green/red } \rightarrow \text { no color: } 7\end{array}$ & $\begin{array}{l}\text { No color } \rightarrow \text { green/red: } 2 \\
\text { Green/red } \rightarrow \text { no color: } 8\end{array}$ \\
\hline EPGR & $\begin{array}{l}\text { No color } \rightarrow \text { green/red: } 1 \\
\text { Green/red } \rightarrow \text { no color: } 2\end{array}$ & $\begin{array}{l}\text { No color } \rightarrow \text { green/red: } 4 \\
\text { Green/red } \rightarrow \text { no color: } 3\end{array}$ & $\begin{array}{l}\text { No color } \rightarrow \text { green/red: } 4 \\
\text { Green/red } \rightarrow \text { no color: } 4\end{array}$ \\
\hline
\end{tabular}

becomes more significant. This result is rather unexpected because for Strategy 3, one waits until the sign of $\hat{\mu}_{i}(n)$ remains unchanged for 3 consecutive days before trading the stock index. This creates a "smoothing effect", reducing the number of times of trading the stock index. Therefore, the dummy variable $R_{3 i}$ fluctuates less frequently than $R_{2 i}$ does. By the same reason as before, Method 3 should show less significant calendar effect than Method 2.

Comparing the results of Table 8 between the securitized real estate and general equity indices, we can see that the six securitized real estate indices show a greater change in number of green and red entries between the three methods, indicating that the difference in the overall calendar effect between the three methods is greater for the six securitized real estate indices. One possible reason for this result is that the general equity indices represent the whole market. They consist of stocks of companies of various businesses, so the stock returns are less correlated and hence more risk is diversified. However, the securitized real estate indices represent the property market, which is only a sector of the whole market. Due to similar nature of business (mainly real estate), the stock returns are more correlated, so less risk is diversified and hence their volatility is higher than that of the general equity indices. Therefore, the Shiryaev-Zhou index of the securitized real estate indices fluctuates more frequently than that of the general equity indices. As a result, when we shift from Strategy 1 to Strategies 2 and 3, the overall calendar effect changes by a larger extent. This implies that by investing in the whole market, we not only benefit from broader diversification, but also maintain a stable calendar effect when the trading strategy shifts from one to another, so the trading rules according to the calendar effects are easier to follow.

\subsection{Halloween effect}

In this part, we apply Methods 1, 2 and 3 described in Section 4 to test the Halloween effect of the 12 stock indices, and use the normal method for comparison. The results are shown in the Tables 9, 10, 11 and 12 .

Note that "p-value" in the above tables represents the $\mathrm{p}$-value calculated by performing a two-tailed z-test, but a one-tailed test is conducted in this study, so the cases where $\hat{\beta}<0$ (or $\hat{\lambda}<0$ ) would be treated as the reverse of the Halloween effect. From Table $9, \hat{\lambda}>0$ for all 12 stock indices. However, only NKY, CAC and DAX show significant statistics at $5 \%$ level. This implies that using linear regression, all 12 stock indices show Halloween effect. However, only the above three indices show significant effect at $5 \%$ level. For each economy except Hong Kong, the general equity index has a smaller p-value of $\hat{\lambda}$ than the securitized real estate index does, indicating a more significant Halloween effect.

However, when applying Methods 1, 2 and 3, there are over half of the cases in which the p-value is below 0.05 (see Tables 10, 11 and 12), showing significant Halloween effect (or the reverse, for cases where $\hat{\beta}<0$ ) at $5 \%$ level. 
Table 9. The test results for the Halloween effect using the normal method

\begin{tabular}{|c|c|c|c|c|c|c|}
\hline Index & HSI & NKY & SPX & UKX & CAC & DAX \\
\hline$\hat{\lambda}$ & 0.000284 & 0.000886 & 0.000431 & 0.000470 & 0.000824 & 0.000900 \\
\hline p-value & 0.513 & 0.028 & 0.183 & 0.136 & 0.034 & 0.026 \\
\hline Index & ELHK & ELJP & UNUS & ELUK & EPFR & EPGR \\
\hline$\hat{\lambda}$ & 0.000621 & 0.000577 & 0.000428 & 0.000356 & 0.000493 & 0.000527 \\
\hline p-value & 0.223 & 0.289 & 0.349 & 0.306 & 0.134 & 0.224 \\
\hline
\end{tabular}

Table 10. The test results for the Halloween effect using Method 1

\begin{tabular}{|c|c|c|c|c|c|c|}
\hline \multicolumn{7}{|c|}{ HSI } \\
\hline $\begin{array}{l}\text { Moving-window } \\
\text { size }\end{array}$ & 40 & 80 & 120 & 160 & 200 & 240 \\
\hline$\hat{\beta}$ & -0.1741 & 0.1244 & 0.3678 & 0.3071 & 0.0225 & -0.2048 \\
\hline $\mathrm{p}$-value & 0.001 & 0.022 & $<0.001$ & $<0.001$ & 0.685 & $<0.001$ \\
\hline \multicolumn{7}{|c|}{ NKY } \\
\hline $\begin{array}{l}\text { Moving-window } \\
\text { size }\end{array}$ & 40 & 80 & 120 & 160 & 200 & 240 \\
\hline$\hat{\beta}$ & 0.3255 & 0.3539 & 0.2478 & -0.0436 & -0.1799 & 0.0278 \\
\hline $\mathrm{p}$-value & $<0.001$ & $<0.001$ & $<0.001$ & 0.420 & 0.001 & 0.608 \\
\hline \multicolumn{7}{|c|}{ SPX } \\
\hline $\begin{array}{l}\text { Moving-window } \\
\text { size }\end{array}$ & 40 & 80 & 120 & 160 & 200 & 240 \\
\hline$\hat{\beta}$ & 0.4908 & 0.5007 & 0.4024 & -0.1287 & -0.2291 & -0.1953 \\
\hline $\mathrm{p}$-value & $<0.001$ & $<0.001$ & $<0.001$ & 0.029 & $<0.001$ & 0.001 \\
\hline \multicolumn{7}{|c|}{ UKX } \\
\hline $\begin{array}{l}\text { Moving-window } \\
\text { size }\end{array}$ & 40 & 80 & 120 & 160 & 200 & 240 \\
\hline$\hat{\beta}$ & 0.4829 & 0.6263 & 0.4260 & -0.0762 & -0.2967 & -0.1004 \\
\hline $\mathrm{p}$-value & $<0.001$ & $<0.001$ & $<0.001$ & 0.170 & $<0.001$ & 0.074 \\
\hline \multicolumn{7}{|c|}{$\mathrm{CAC}$} \\
\hline $\begin{array}{l}\text { Moving-window } \\
\text { size }\end{array}$ & 40 & 80 & 120 & 160 & 200 & 240 \\
\hline$\hat{\beta}$ & 0.4469 & 0.5425 & 0.3392 & -0.0883 & -0.1629 & 0.0436 \\
\hline p-value & $<0.001$ & $<0.001$ & $<0.001$ & 0.110 & 0.004 & 0.442 \\
\hline \multicolumn{7}{|c|}{ DAX } \\
\hline $\begin{array}{l}\text { Moving-window } \\
\text { size }\end{array}$ & 40 & 80 & 120 & 160 & 200 & 240 \\
\hline$\hat{\beta}$ & 0.6362 & 0.5585 & 0.1475 & -0.1901 & -0.2813 & -0.0029 \\
\hline $\mathrm{p}$-value & $<0.001$ & $<0.001$ & 0.009 & 0.001 & $<0.001$ & 0.960 \\
\hline \multicolumn{7}{|c|}{ ELHK } \\
\hline $\begin{array}{l}\text { Moving-window } \\
\text { size }\end{array}$ & 40 & 80 & 120 & 160 & 200 & 240 \\
\hline$\hat{\beta}$ & -0.2094 & 0.1284 & 0.3600 & 0.1581 & 0.1708 & -0.0914 \\
\hline $\mathrm{p}$-value & $<0.001$ & 0.018 & $<0.001$ & 0.004 & 0.002 & 0.099 \\
\hline \multicolumn{7}{|c|}{ ELJP } \\
\hline $\begin{array}{l}\text { Moving-window } \\
\text { size } \\
\end{array}$ & 40 & 80 & 120 & 160 & 200 & 240 \\
\hline$\hat{\beta}$ & 0.2446 & 0.1114 & -0.2608 & -0.5376 & -0.3684 & -0.0914 \\
\hline $\mathrm{p}$-value & $<0.001$ & 0.039 & $<0.001$ & $<0.001$ & $<0.001$ & 0.099 \\
\hline
\end{tabular}


End of Table 10

\begin{tabular}{|c|c|c|c|c|c|c|}
\hline \multicolumn{7}{|c|}{ UNUS } \\
\hline $\begin{array}{l}\text { Moving-window } \\
\text { size }\end{array}$ & 40 & 80 & 120 & 160 & 200 & 240 \\
\hline$\hat{\beta}$ & 0.2139 & 0.0695 & -0.0758 & -0.3018 & -0.3892 & -0.3786 \\
\hline $\mathrm{p}$-value & $<0.001$ & 0.212 & 0.184 & $<0.001$ & $<0.001$ & $<0.001$ \\
\hline \multicolumn{7}{|c|}{ ELUK } \\
\hline $\begin{array}{l}\text { Moving-window } \\
\text { size }\end{array}$ & 40 & 80 & 120 & 160 & 200 & 240 \\
\hline$\hat{\beta}$ & 0.1570 & -0.0380 & -0.1234 & -0.3018 & -0.0935 & 0.0371 \\
\hline $\mathrm{p}$-value & 0.004 & 0.491 & 0.026 & $<0.001$ & 0.094 & 0.503 \\
\hline \multicolumn{7}{|c|}{ EPFR } \\
\hline $\begin{array}{l}\text { Moving-window } \\
\text { size }\end{array}$ & 40 & 80 & 120 & 160 & 200 & 240 \\
\hline$\hat{\beta}$ & 0.4485 & 0.3415 & 0.1454 & -0.3156 & -0.4945 & -0.3342 \\
\hline p-value & $<0.001$ & $<0.001$ & 0.010 & $<0.001$ & $<0.001$ & $<0.001$ \\
\hline \multicolumn{7}{|c|}{ EPGR } \\
\hline $\begin{array}{l}\text { Moving-window } \\
\text { size }\end{array}$ & 40 & 80 & 120 & 160 & 200 & 240 \\
\hline$\hat{\beta}$ & 0.0097 & 0.0147 & -0.1283 & -0.1373 & -0.0518 & 0.0603 \\
\hline $\mathrm{p}$-value & 0.858 & 0.786 & 0.018 & 0.012 & 0.340 & 0.266 \\
\hline
\end{tabular}

Table 11. The test results for the Halloween effect using Method 2

\begin{tabular}{|c|c|c|c|c|c|c|}
\hline \multicolumn{7}{|c|}{ HSI } \\
\hline $\begin{array}{l}\text { Moving-window } \\
\text { size }\end{array}$ & 40 & 80 & 120 & 160 & 200 & 240 \\
\hline$\hat{\beta}$ & -0.1771 & 0.1409 & 0.3362 & 0.3176 & 0.0103 & -0.2003 \\
\hline $\mathrm{p}$-value & 0.001 & 0.010 & $<0.001$ & $<0.001$ & 0.856 & $<0.001$ \\
\hline \multicolumn{7}{|c|}{ NKY } \\
\hline $\begin{array}{l}\text { Moving-window } \\
\text { size }\end{array}$ & 40 & 80 & 120 & 160 & 200 & 240 \\
\hline$\hat{\beta}$ & 0.3091 & 0.3523 & 0.2376 & -0.0405 & -0.2034 & 0.0409 \\
\hline $\mathrm{p}$-value & $<0.001$ & $<0.001$ & $<0.001$ & 0.406 & $<0.001$ & 0.450 \\
\hline \multicolumn{7}{|c|}{ SPX } \\
\hline $\begin{array}{l}\text { Moving-window } \\
\text { size }\end{array}$ & 40 & 80 & 120 & 160 & 200 & 240 \\
\hline$\hat{\beta}$ & 0.4931 & 0.4694 & 0.3941 & -0.1464 & -0.2460 & -0.2049 \\
\hline $\mathrm{p}$-value & $<0.001$ & $<0.001$ & $<0.001$ & 0.013 & $<0.001$ & 0.001 \\
\hline \multicolumn{7}{|c|}{ UKX } \\
\hline $\begin{array}{l}\text { Moving-window } \\
\text { size } \\
\end{array}$ & 40 & 80 & 120 & 160 & 200 & 240 \\
\hline$\hat{\beta}$ & 0.4825 & 0.6253 & 0.4124 & -0.0962 & -0.2999 & -0.0973 \\
\hline $\mathrm{p}$-value & $<0.001$ & $<0.001$ & $<0.001$ & 0.083 & $<0.001$ & 0.084 \\
\hline \multicolumn{7}{|c|}{ CAC } \\
\hline $\begin{array}{l}\text { Moving-window } \\
\text { size }\end{array}$ & 40 & 80 & 120 & 160 & 200 & 240 \\
\hline$\hat{\beta}$ & 0.4102 & 0.5291 & 0.3312 & -0.0974 & -0.1708 & 0.0468 \\
\hline p-value & $<0.001$ & $<0.001$ & $<0.001$ & 0.078 & 0.002 & 0.410 \\
\hline \multicolumn{7}{|c|}{ DAX } \\
\hline $\begin{array}{l}\text { Moving-window } \\
\text { size }\end{array}$ & 40 & 80 & 120 & 160 & 200 & 240 \\
\hline$\hat{\beta}$ & 0.6391 & 0.5467 & 0.1223 & -0.2068 & -0.2775 & -0.0029 \\
\hline $\mathrm{p}$-value & $<0.001$ & $<0.001$ & 0.030 & $<0.001$ & $<0.001$ & 0.960 \\
\hline
\end{tabular}


End of Table 11

\begin{tabular}{|c|c|c|c|c|c|c|}
\hline \multicolumn{7}{|c|}{ ELHK } \\
\hline $\begin{array}{l}\text { Moving-window } \\
\text { size }\end{array}$ & 40 & 80 & 120 & 160 & 200 & 240 \\
\hline$\hat{\beta}$ & -0.2094 & 0.1343 & 0.3580 & 0.1610 & 0.1918 & -0.0929 \\
\hline p-value & $<0.001$ & 0.013 & $<0.001$ & 0.003 & $<0.001$ & 0.093 \\
\hline \multicolumn{7}{|c|}{ ELJP } \\
\hline $\begin{array}{l}\text { Moving-window } \\
\text { size }\end{array}$ & 40 & 80 & 120 & 160 & 200 & 240 \\
\hline$\hat{\beta}$ & 0.2358 & 0.0734 & -0.2873 & -0.5479 & -0.3551 & -0.1182 \\
\hline $\mathrm{p}$-value & $<0.001$ & 0.175 & $<0.001$ & $<0.001$ & $<0.001$ & 0.029 \\
\hline \multicolumn{7}{|c|}{ UNUS } \\
\hline $\begin{array}{l}\text { Moving-window } \\
\text { size } \\
\end{array}$ & 40 & 80 & 120 & 160 & 200 & 240 \\
\hline$\hat{\beta}$ & 0.2105 & 0.0431 & -0.0726 & -0.3057 & -0.4076 & -0.3833 \\
\hline $\mathrm{p}$-value & $<0.001$ & 0.439 & 0.203 & $<0.001$ & $<0.001$ & $<0.001$ \\
\hline \multicolumn{7}{|c|}{ ELUK } \\
\hline $\begin{array}{l}\text { Moving-window } \\
\text { size } \\
\end{array}$ & 40 & 80 & 120 & 160 & 200 & 240 \\
\hline$\hat{\beta}$ & 0.1447 & -0.0501 & -0.1219 & -0.3153 & -0.0966 & 0.0402 \\
\hline $\mathrm{p}$-value & 0.008 & 0.364 & 0.027 & $<0.001$ & 0.084 & 0.467 \\
\hline \multicolumn{7}{|c|}{ EPFR } \\
\hline $\begin{array}{l}\text { Moving-window } \\
\text { size } \\
\end{array}$ & 40 & 80 & 120 & 160 & 200 & 240 \\
\hline$\hat{\beta}$ & 0.4542 & 0.3335 & 0.1214 & -0.3063 & -0.5021 & -0.3116 \\
\hline $\mathrm{p}$-value & $<0.001$ & $<0.001$ & 0.032 & $<0.001$ & $<0.001$ & $<0.001$ \\
\hline \multicolumn{7}{|c|}{ EPGR } \\
\hline $\begin{array}{l}\text { Moving-window } \\
\text { size } \\
\end{array}$ & 40 & 80 & 120 & 160 & 200 & 240 \\
\hline$\hat{\beta}$ & 0.0051 & 0.0176 & -0.1387 & -0.1507 & -0.0709 & 0.0456 \\
\hline $\mathrm{p}$-value & 0.924 & 0.745 & 0.011 & 0.006 & 0.191 & 0.401 \\
\hline
\end{tabular}

Table 12. The test results for the Halloween effect using Method 3

\begin{tabular}{|c|c|c|c|c|c|c|}
\hline \multicolumn{7}{|c|}{ HSI } \\
\hline $\begin{array}{l}\text { Moving-window } \\
\text { size }\end{array}$ & 40 & 80 & 120 & 160 & 200 & 240 \\
\hline$\hat{\beta}$ & -0.1993 & 0.1439 & 0.3472 & 0.3095 & -0.0080 & -0.2112 \\
\hline $\mathrm{p}$-value & 0.001 & 0.008 & $<0.001$ & $<0.001$ & 0.886 & $<0.001$ \\
\hline \multicolumn{7}{|c|}{ NKY } \\
\hline $\begin{array}{l}\text { Moving-window } \\
\text { size }\end{array}$ & 40 & 80 & 120 & 160 & 200 & 240 \\
\hline$\hat{\beta}$ & 0.2901 & 0.3362 & 0.2113 & -0.0407 & -0.1710 & 0.0483 \\
\hline $\mathrm{p}$-value & $<0.001$ & $<0.001$ & $<0.001$ & 0.452 & 0.002 & 0.372 \\
\hline \multicolumn{7}{|c|}{ SPX } \\
\hline $\begin{array}{l}\text { Moving-window } \\
\text { size }\end{array}$ & 40 & 80 & 120 & 160 & 200 & 240 \\
\hline$\hat{\beta}$ & 0.4444 & 0.4959 & 0.3742 & -0.1882 & -0.2271 & -0.1828 \\
\hline $\mathrm{p}$-value & $<0.001$ & $<0.001$ & $<0.001$ & 0.001 & $<0.001$ & 0.003 \\
\hline \multicolumn{7}{|c|}{ UKX } \\
\hline $\begin{array}{l}\text { Moving-window } \\
\text { size }\end{array}$ & 40 & 80 & 120 & 160 & 200 & 240 \\
\hline$\hat{\beta}$ & 0.5098 & 0.6481 & 0.4191 & -0.1456 & -0.2629 & -0.1004 \\
\hline p-value & $<0.001$ & $<0.001$ & $<0.001$ & 0.009 & $<0.001$ & 0.074 \\
\hline
\end{tabular}


End of Table 12

\begin{tabular}{|c|c|c|c|c|c|c|}
\hline \multicolumn{7}{|c|}{ CAC } \\
\hline $\begin{array}{l}\text { Moving-window } \\
\text { size }\end{array}$ & 40 & 80 & 120 & 160 & 200 & 240 \\
\hline$\hat{\beta}$ & 0.4116 & 0.5265 & 0.3249 & -0.1210 & -0.1696 & 0.0404 \\
\hline $\mathrm{p}$-value & $<0.001$ & $<0.001$ & $<0.001$ & 0.065 & 0.003 & 0.476 \\
\hline \multicolumn{7}{|c|}{ DAX } \\
\hline $\begin{array}{l}\text { Moving-window } \\
\text { size }\end{array}$ & 40 & 80 & 120 & 160 & 200 & 240 \\
\hline$\hat{\beta}$ & 0.6499 & 0.5469 & 0.1128 & -0.2099 & -0.2876 & 0.0072 \\
\hline $\mathrm{p}$-value & $<0.001$ & $<0.001$ & 0.046 & $<0.001$ & $<0.001$ & 0.901 \\
\hline \multicolumn{7}{|c|}{ ELHK } \\
\hline $\begin{array}{l}\text { Moving-window } \\
\text { size }\end{array}$ & 40 & 80 & 120 & 160 & 200 & 240 \\
\hline$\hat{\beta}$ & -0.2256 & 0.1283 & 0.3240 & 0.1581 & 0.1816 & -0.1051 \\
\hline $\mathrm{p}$-value & $<0.001$ & 0.018 & $<0.001$ & 0.004 & 0.001 & 0.057 \\
\hline \multicolumn{7}{|c|}{ ELJP } \\
\hline $\begin{array}{l}\text { Moving-window } \\
\text { size }\end{array}$ & 40 & 80 & 120 & 160 & 200 & 240 \\
\hline$\hat{\beta}$ & 0.2460 & 0.0544 & -0.3208 & -0.5507 & -0.3654 & -0.0949 \\
\hline $\mathrm{p}$-value & $<0.001$ & 0.314 & $<0.001$ & $<0.001$ & $<0.001$ & 0.079 \\
\hline \multicolumn{7}{|c|}{ UNUS } \\
\hline $\begin{array}{l}\text { Moving-window } \\
\text { size }\end{array}$ & 40 & 80 & 120 & 160 & 200 & 240 \\
\hline$\hat{\beta}$ & 0.2178 & 0.0339 & -0.0693 & -0.3233 & -0.3872 & -0.3964 \\
\hline p-value & $<0.001$ & 0.542 & 0.224 & $<0.001$ & $<0.001$ & $<0.001$ \\
\hline \multicolumn{7}{|c|}{ ELUK } \\
\hline $\begin{array}{l}\text { Moving-window } \\
\text { size }\end{array}$ & 40 & 80 & 120 & 160 & 200 & 240 \\
\hline$\hat{\beta}$ & 0.1709 & -0.0516 & -0.1309 & -0.3065 & -0.0888 & 0.0265 \\
\hline $\mathrm{p}$-value & 0.002 & 0.350 & 0.018 & $<0.001$ & 0.112 & 0.632 \\
\hline \multicolumn{7}{|c|}{ EPFR } \\
\hline $\begin{array}{l}\text { Moving-window } \\
\text { size }\end{array}$ & 40 & 80 & 120 & 160 & 200 & 240 \\
\hline$\hat{\beta}$ & 0.4962 & 0.3170 & 0.0831 & -0.2987 & -0.5204 & -0.3093 \\
\hline $\mathrm{p}$-value & $<0.001$ & $<0.001$ & 0.142 & $<0.001$ & $<0.001$ & $<0.001$ \\
\hline \multicolumn{7}{|c|}{ EPGR } \\
\hline $\begin{array}{l}\text { Moving-window } \\
\text { size }\end{array}$ & 40 & 80 & 120 & 160 & 200 & 240 \\
\hline$\hat{\beta}$ & -0.0226 & 0.0381 & -0.1490 & -0.1433 & -0.0709 & 0.0778 \\
\hline $\mathrm{p}$-value & 0.676 & 0.482 & 0.006 & 0.009 & 0.191 & 0.152 \\
\hline
\end{tabular}

This reveals that additional channels of calendar effects are detected. In addition, Methods 1, 2 and 3 show the following similarities to Hui and Chan (2016)'s results:

1 . For the 6 general equity indices, if the moving-window size is small $(<=120)$, then for almost all the cases, $\hat{\beta}>0$ and the p-value is below 0.05 , revealing that the Halloween effect is highly significant. However, if the moving-window size is large $(>=160)$, then $\hat{\beta}<0$ for more than half of the cases, and the $\mathrm{p}$-value is below 0.05 for slight majority of cases, reflecting that for the majority of the cases, the Halloween effect goes into reverse, but the effect is not so significant.
2. For the 6 securitized real estate indices, there are slight majority of the cases where $\hat{\beta}<0$, i.e. the Halloween effect goes into reverse. In particular, for larger moving-window sizes $(>=160)$, the cases where $\beta<0$ dominate.

In particular, for the 4 western general equity indices (SPX, UKX, CAC and DAX) with moving-window size 120 or smaller, $\hat{\beta}>0$ and the $p$-value is below 0.05 , showing that the Halloween effect is highly significant. This is natural because the most of the previous studies on Halloween effect work on equity markets of European and North American countries (see Section 1), so the four 
western general equity indices behave normally. However, most Asian markets are still at a developing stage, so their calendar effects may not be the same as those of the European and North America markets. Investors should be aware of this phenomenon when investing in those developing markets. The small moving-window size means that the "delaying effect" (see Hui and Chan, 2016 for detail explanation) is not so significant that the calendar effect is not distorted.

However, comparing the results between Tables 8, 9 and 10 , we can see that there is only a slight difference in the number of cases where $\hat{\beta}<0$ between Methods 1 , 2 and 3. This reveals that the patterns of Halloween effect shown by the three methods are almost the same. The main reason is that Methods 2 and 3 are based on Strategies 2 and 3 respectively. For Strategies 2 and 3, an investor waits until the sign of $\hat{\mu}_{i}(n)$ remains unchanged for 2 and 3 consecutive days respectively before trading the stock index. Comparing with Strategy 1, this is a small change only. Hence the difference in the overall calendar effect shown by the three methods in Tables 5, 6 and 7 is not so significant. The Halloween effect concerns with the hypotheses $H_{11}, H_{21}$ and $H_{31}$, which say that the "holding periods" of Strategy 1, 2 and 3, respectively (i.e. the percentage of time of which $R_{1 i}=1, R_{2 i}=1$ and $R_{3 i}=1$ respectively), are significantly longer from November to April than from May to October. Taking the average values of $R_{1 i}, R_{2 i}$ and $R_{3 i}$ in six months, this would lower the standard deviation of the average values of $R_{1 i}, R_{2 i}$ and $R_{3 i}$. Therefore, the difference in patterns of Halloween effect shown by the three methods is very small.

\section{Conclusion}

In this study, we construct two new tests of calendar effects based on Hui and Chan (2018c)'s two new strategies of which after $\hat{\mu}_{i}(n)$ changes sign, one waits until $\hat{\mu}_{i}(n)$ remains at the same sign for two or three consecutive days before buying or selling the stock index (called Methods 2 and 3 respectively in this study). we apply these two methods on 12 stock indices during 1996-2016, and compare the results with that using Hui and Chan (2016)'s method (Method 1). The major results are as follows:

(1) When the moving-window size increases, the calendar effects generally diminish.

(2) Significant January and Halloween effects exist in most of the general equity indices (especially for the 4 western economies for smaller movingwindow sizes). However, the two effects are less prevalent or even go into reverse sometimes for the securitized real estate indices.

(3) Method 2 shows less significant calendar effect than Method 1 does, but Method 3 shows more significant calendar effect than Method 2 does.

(4) The securitized real estate indices show a greater difference in the overall calendar effect between the three methods than the general equity indices do.
The common advantage of the three methods is that they can show the percentage of time of which the stock index is held in a month according to a trading strategy which outperforms "buy-and-hold" in general. However, Method 1 is based on Strategy 1, of which Hui and Chan (2018c) find that the strategy underperforms "buy-and-hold" for slight majority of the cases. Since Hui and Chan (2018c) find two strategies (Strategies 2 and 3 in this study) which yield greater profit than Strategy 1 does, we construct two new tests of calendar effects which can show the length of "holding periods" of Strategies 2 and 3 respectively. From the results of our new methods (Methods 2 and 3), we can know in which months, the stock indices should be held for a significantly longer period of time than average, according to Strategies 2 and 3. Hence our method is superior to Hui and Chan (2016)'s method. In addition, the result that the securitized real estate indices show a greater difference in the overall calendar effect between the three methods than the general equity indices do has meaningful implications. The general equity indices represent the whole market. Changing the trading strategy does not alter the calendar effect a lot. However, for a certain sector of the whole market like real estate, the volatility is larger (see Sub-section 5.1 for explanation) and hence shifting the trading strategy would alter the calendar effect by a larger extent. Therefore, investors should be aware of this risk when investing on a certain sector of the market. However, for the whole market, the risk is smaller due to broader diversification. Hence it is more suitable for investors who are only able to bear smaller risk. Furthermore, we find that if the moving-window size is small, both January and Halloween effects are significant for the 4 western general equity indices. This reflects that most of the previous studies on Halloween and January effects work on equity markets of European and North American countries (see Section 1), so the four western general equity indices behave normally. The small moving-window size means that the "delaying effect" (see Hui and Chan, 2016 for detail explanation) is not so significant that the calendar effect is not distorted. Therefore, if investors adopt Strategies 1, 2 or 3, and use a small moving-window size to invest in European and North American equity markets, they can still rely on calendar effects to a certain extent. However, for larger moving-window sizes/Asian markets/ securitized real estate markets, those traditional calendar effects may not necessarily hold. Larger moving-window sizes increases the "delaying effect" and hence distorts the calendar effect. Most Asian markets are still at a developing stage, so their calendar effects may not be the same as those of the European and North America markets. The securitized real estate market is just a sector of the whole market, so its calendar effect may be different from that of the whole market, too. Investors should be aware of this phenomenon in order to earn more profits. One may construct a trading strategy of which an investor waits until the sign of $\hat{\mu}_{i}(n)$ remains unchanged for 4 or more consecutive days before trading the stock/stock index, and construct a new test of calendar effect according to this strategy. This is a possible scope of future research. 


\section{Acknowledgement}

We are grateful for the financial support from the PolyU Internal Research Grants (Project \# G-YBJL and G-YBQ8).

\section{Reference}

Agrawal, A., \& Tandon, K. (1994). Anomalies or illusions? Evidence from stock markets in eighteen countries. Journal of International Money and Finance, 13(1), 83-106. https://doi.org/10.1016/0261-5606(94)90026-4

Almudhaf, F., \& Hansz, J. (2011). Systematic equity return patterns in listed European property companies. International Real Estate Review, 14(1), 61-84.

Andrade, S., Chhaochharia, V., \& Fuerst, M. (2012). "Sell in May and go away" just won't go away. Available at SSRN 2115197. https://doi.org/10.2139/ssrn.2115197

Barber, B. M., \& Odean, T. (2000). Trading is hazardous to your wealth: the common stock investment performance of individual investors. Journal of Finance, 55(2), 773-806.

https://doi.org/10.1111/0022-1082.00226

Bouman, S., \& Jacobsen, B. (2002). The Halloween indicator, "Sell in May and go away": another puzzle. The American Economic Review, 92(5), 1618-1635. https://doi.org/10.1257/000282802762024683

Brounen, D., \& Ben-Hamo, Y. (2009). Calendar anomalies: the case of international property shares. Journal of Real Estate Finance and Economics, 38(2), 115-136.

https://doi.org/10.1007/s11146-007-9088-9

Cheung, K. C., \& Coutts, J. A. (1999). The January effect and monthly seasonality in the Hang Seng index: 1985-97. Applied Economics Letters, 6(2), 121-123.

https://doi.org/10.1080/135048599353753

Du Toit, J., \& Peskir, G. (2008). Selling a stock at the ultimate maximum. The Annals of Applied Probability, 19, 983-1014. https://doi.org/10.1214/08-AAP566

Fountas, S., \& Segredakis, K. N. (2002). Emerging stock markets return seasonalities: the January effect and the tax-loss selling hypothesis. Applied Financial Economics, 12(4), 291-299. https://doi.org/10.1080/09603100010000839

$\mathrm{Gu}$, A. Y. (2003). The declining January effect: evidences from the US equity markets. The Quarterly Review of Economics and Finance, 43(2), 395-404.

https://doi.org/10.1016/S1062-9769(02)00160-6

Hansen, P. (2005). A test for superior predictive ability. Journal of Business \& Economic Statistics, 23(4), 365-380. https://doi.org/10.1198/073500105000000063

Hansen, P. R., Lunde, A., \& Nason, J. M. (2005). Testing the significance of Calendar Effects. Federal Reserve Bank of Atlanta Working Paper, (2005-02). https://doi.org/10.2139/ssrn.388601

Hardin III, W., Liano, K., \& Huang, G. (2005). Real estate investment trusts and calendar anomalies: revisited. International Real Estate Review, 8(1), 83-94.

Hui, E. C. M., \& Chan, K. K. K. (2014). The global financial crisis: is there any contagion between real estate and equity markets?. Physica A: Statistical Mechanics and its Applications, 405, 216-225. https://doi.org/10.1016/j.physa.2014.03.008

Hui, E. C. M., \& Chan, K. K. K. (2015). Testing calendar effects on global securitized real estate markets by Shiryaev-Zhou index. Habitat International, 48, 38-45.

https://doi.org/10.1016/j.habitatint.2015.03.009

Hui, E. C. M., \& Chan, K. K. K. (2016). Testing calendar effects of international equity and real estate markets. Journal of Real Estate Finance and Economics, 56(1), 140-158.
Hui, E. C. M., \& Chan, K. K. K. (2018a). A new time-dependent trading strategy for securitized real estate and equity indices. International Journal of Strategic Property Management, 22(1), 64-79. https://doi.org/10.3846/1648715X.2016.1260072

Hui, E. C. M., \& Chan, K. K. K. (2018b). Optimal trading strategy during bull and bear markets. International Journal of Strategic Property Management, forthcoming.

Hui, E. C. M., \& Chan, K. K. K. (2018c). New trading strategies to increase profit by "smoothing effect". Working paper.

Hui, E. C. M., Wright, J. A., \& Yam, S. C. P. (2014a). Calendar effects and real estate securities. Journal of Real Estate Finance and Economics, 49(1), 91-115. https://doi.org/10.1007/s11146-012-9398-4

Hui, E. C. M., Yam, S. C. P, Wright, J., \& Chan, K. K. K. (2014b). Shall we buy and hold? Evidence from Asian real estate markets. Journal of Property Investment and Finance, 32(2), 168186. https://doi.org/10.1108/JPIF-09-2013-0059

Jacobsen, B., \& Visaltanachoti, N. (2009). The Halloween effect in US sectors. Financial Review, 44(3), 437-459.

https://doi.org/10.1111/j.1540-6288.2009.00224.x

Kang, S. H., Jiang, Z., Lee, Y., \& Yoon, S. M. (2010). Weather effects on the returns and volatility of the Shanghai stock market. Physica A: Statistical Mechanics and its Applications, 389(1), 91-99. https://doi.org/10.1016/j.physa.2009.09.010

Keim, D. B. (1983). Size-related anomalies and stock return seasonality: further empirical evidence. Journal of Financial Economics, 12(1), 13-32. https://doi.org/10.1016/0304-405X(83)90025-9

Lean, H. H. (2011). The Halloween puzzle in selected Asian stock markets. International Journal of Economics and Management, 5, 216-225.

Lucey, B. M., \& Whelan, S. F. (2002). A promising timing strategy in equity markets. Journal of the Statistical and Social Inquiry Society of Ireland, 31, 74-110.

Maberly, E. D., \& Pierce, R. (2004). Stock market efficiency withstands another challenge: solving the "sell in May/buy after Halloween" puzzle. Econ Journal Watch, 1(1), 29-46.

Malkiel, B. G., \& Fama, E. F. (1970). Efficient capital markets: a review of theory and empirical work. Journal of Finance, 25(2), 383-417. https://doi.org/10.1111/j.1540-6261.1970.tb00518.x

Shiryaev, A. N., Xu, Z., \& Zhou, X. Y. (2008). Thou shalt buy and hold. Quantitative Finance, 8(8), 765-776. https://doi.org/10.1080/14697680802563732

Sullivan, R., Timmermann, A., \& White, H. (2001). Dangers of data mining: the case of calendar effects in stock returns. Journal of Econometrics, 105(1), 249-286. https://doi.org/10.1016/S0304-4076(01)00077-X

Wachtel, S. B. (1942). Certain observations on seasonal movements in stock prices. The Journal of Business of the University of Chicago, 15(2), 184-193. https://doi.org/10.1086/232617

Wong, W. K., Wright, J. A., Yam, S. C. P., \& Yung, S. P. (2012). A mixed Sharpe ratio. Risk and Decision Analysis, 3, 37-65.

White, H. (2000). A reality check for data snooping. Econometrica, 68(5), 1097-1126. https://doi.org/10.1111/1468-0262.00152

Yam, S. C. P., Yung, S. P., \& Zhou, W. (2012a). A unified 'bangbang' principle with respect to R-invariant performance benchmarks. Theory of Probability and Its Applications, 57(2), 405-414. https://doi.org/10.4213/tvp4457

Yam, S. C. P., Yung, S. P., \& Zhou, W. (2012b). Optimal selling time in stock market over a finite time horizon. Acta Mathematicae Applicatae Sinica, 28(3), 557-570. https://doi.org/10.1007/s10255-012-0169-Z

Yam, S. C. P., Yung, S. P., \& Zhou, W. (2009). Two rationales behind 'buy-and-hold or sell-at-once'. Journal of Applied Probability, 46, 651-668. https://doi.org/10.1239/jap/1253279844 